\title{
Modulatory mechanisms in Pavlovian conditioning
}

\author{
DALE SWARTZENTRUBER \\ Ohio Wesleyan University, Delaware, Ohio
}

\begin{abstract}
Pavlovian conditioning has traditionally been thought to involve the acquisition of excitatory and inhibitory associations between a conditioned stimulus (CS) and an unconditioned stimulus (US). Recent research, however, has encouraged the view that Pavlovian learning may also encompass a higher order modulatory mechanism, in which animals use information about another stimulus to control responding to the CS. Positive modulators signal a positive relationship between the CS and the US, whereas negative modulators signal that the CS-US relationship is not in force. In both cases, the modulatory control appears to be orthogonal to the modulator's direct associations with the US. This article reviews and evaluates the literature on this Pavlovian modulatory mechanism.
\end{abstract}

In his analysis of the role that a stimulus plays in operant responding, Skinner (1938) recognized that in many situations, responses are reinforced only in a particular stimulus environment. On the basis of these environmental cues, the organism comes to discriminate the occasions on which responses are reinforced from those in which responses are not reinforced:

\begin{abstract}
Although the response is free to come about in a very large number of stimulating situations, it will be effective in producing a reinforcement only in a small part of them. The favorable situation is usually marked in some way, and the organism makes a discrimination ... It comes to respond whenever a stimulus is present which has been present upon the occasion of a previous reinforcement and not to respond otherwise. The prior stimulus does not elicit the response; it merely sets the occasion upon which the response will be reinforced. (p. 178)
\end{abstract}

Skinner suggested that this is the manner in which responses become restricted to specific stimulus situations. As a result, the presence of the stimulus effectively modulates the response.

Pavlovian conditioning, on the other hand, is typically understood in terms of learned binary associations between stimuli. Animals acquire excitatory and inhibitory associations as a function of the predictive relationship that exists between conditioned stimuli (CSs) and unconditioned stimuli (USs). Perhaps because even the learning of quite complicated discriminations can be accounted for in terms of these simple associations, theories of Pavlovian conditioning have largely ignored the

Preparation of this article was partially supported by National Institute of Mental Health postdoctoral training Grant MH 09896 to the author. I thank Peter C. Holland, Robert A. Rescorla, and anonymous reviewers for their comments on the manuscript. Address correspondence to the author, Department of Psychology, Ohio Wesleyan University, Delaware, $\mathrm{OH} 43015$ (e-mail:deswartz@cc.owu.edu).

-Accepted by previous editor, Vincent M. LoLordo potential for a modulatory stimulus role analogous to that described by Skinner for operant paradigms.

However, recent research from several laboratories has shown that stimuli do appear to modulate conditioned responding to other stimuli. Researchers have provided evidence that an animal can learn that one stimulus provides information about a second stimulus' contingency with the US. The probability of responding to the second stimulus comes to depend on the presence of the modulatory stimulus. This role appears to be relatively independent of the modulator's own associations with the US.

There has been a major trend in Pavlovian conditioning research toward examining this modulatory mechanism. The purpose of the present article is to review this research and to describe and evaluate the several existing views on the nature of modulation.

\section{EVIDENCE FOR MODULATION}

Much of the recent interest in the modulatory function of stimuli in Pavlovian conditioning can be traced to the findings of Ross and Holland (1981). They began a systematic investigation of modulation by using appetitive feature-positive, or $\mathrm{AB}+, \mathrm{B}-$, discrimination training in rat subjects. In their experiments, a 5-sec auditory target stimulus (B) was presented on all trials but was reinforced with a food-pellet US only when B was presented in compound with a 5-sec visual stimulus (A). As the discrimination was acquired, responding became greater during $\mathrm{AB}$ compound trials than when $\mathrm{B}$ was presented alone. Solution of such a feature-positive discrimination has typically been thought to involve the acquisition of simple excitatory associations between $\mathrm{A}$ and the US (e.g., Hearst, 1978). However, the results of several procedural manipulations led Ross and Holland to conclude that the visual A stimulus did not always act solely as an excitor, but sometimes served to "set the occasion" for the excitatory B-US association. 
Ross and Holland's (1981) preparation exploited earlier work by Holland (1977), who showed that excitatory appetitive visual and auditory stimuli evoke different response forms. A light that has been paired with food will evoke rearing and orienting toward the food magazine, whereas an auditory stimulus such as a tone or white noise will evoke rapid headjerk responses. Thus, they could assess the specific excitatory associations that are acquired during training by observing the form of the rat's responding during the light and tone.

Using this technique, Ross and Holland (1981) found that the manner in which the feature positive discrimination was solved depended on the temporal relationship between the light and tone on $\mathrm{AB}+$ compound trials. If the light and tone were presented simultaneously, the compound evoked a rearing response, indicating that the light feature, A, had become excitatory. This finding was consistent with prior research and was predicted by a simple associative account; the light was a more valid predictor of the US than was the tone. However, if the light preceded the tone on $\mathrm{AB}+$ compound trials, the rats both reared to the light and headjerked to the tone, suggesting that the tone, as well as the light, was now excitatory. Importantly, the rats only headjerked to the tone when it followed the light. The light thus appeared to have acquired the ability to modulate or set the occasion for the expression of the excitatory tone-US association.

Rescorla (e.g., 1985) has provided comparable data from a pigeon autoshaping preparation. As with Ross and Holland's (1981) preparation, this research exploited the benefits of using stimuli that evoke different excitatory response forms. In pigeons, a keylight stimulus that has been paired with food will come to elicit directed pecking at the key. A diffuse excitatory stimulus, on the other hand, such as white noise, a tone, or an overhead houselight, tends to evoke general activity. In Rescorla's typical feature-positive procedure, a 5-sec keylight target, B, is nonreinforced when presented alone and is reinforced with grain when presented during the last $5 \mathrm{sec}$ of a 15 sec diffuse feature, A. The pigeons rapidly come to respond to the keylight on $\mathrm{AB}+$ compound trials, but not when the keylight is presented alone, $\mathrm{B}-$. Because responding on compound trials takes the form of keypecking, and not simply an increase in general activity, these results again suggest that the auditory feature had not simply become associated with food, but instead modulated or "facilitated" the response to the visual keylight target.

The feature-positive procedure used in these investigations of positive modulation is procedurally the converse of the familiar feature-negative, or $\mathrm{AB}-, \mathrm{B}+$, procedure often used to establish Pavlovian conditioned inhibition to the A feature. The nature of the inhibitory learning that occurs in the feature-negative procedure has received considerable attention over the past two decades. Recent work from several laboratories has suggested that more than one type of inhibitory mechanism may exist; as with positive modulation, the mechanism involved might depend on the temporal relationship between the feature and the target. Holland and his collaborators, for example, have conducted a number of investigations examining a possible role for negative modulation in learning that parallels that of positive modulation (e.g., Holland, 1984, 1985, 1989b; Holland \& Lamarre, 1984). Their work has suggested that inhibition might come about either by acting directly on the US representation, in a manner opposite to simple excitation, or by actively modulating the expression of excitatory CS-US associations. Rescorla (e.g., 1985; see also Jenkins, 1985) has conducted a number of autoshaping experiments to examine inhibitory control and has also provided evidence for a negative modulatory process. In general, considerable evidence now suggests that the traditional view of inhibition as the direct opposite of excitation may need to be reevaluated. At least under some circumstances, inhibitory control can function through a modulatory mechanism that is distinct from simple associations.

Over the past 10 years, the research on Pavlovian modulation has focused on various aspects of the nature of the modulatory learning mechanism. This review will begin by examining the role that excitation plays in positive and negative modulation. The next section will describe the several different views on the nature of the modulatory mechanism and will evaluate the research that has provided evidence for the views. The final section will examine the conditions currently thought to be important for acquisition of modulation.

\section{THE ROLE OF EXCITATORY ASSOCIATIONS IN MODULATORY CONTROL}

Modulatory processes have received attention because their role in learning is not encompassed by our current associative models of Pavlovian conditioning. Positive modulators have properties that appear to transcend their simple associations with other stimuli or with the US. Similarly, the control exerted by negative modulators often appears surprisingly unaffected by their observable excitation. Research on both modulatory processes has focused largely on the extent to which the modulatory functions are independent of excitatory associations.

\section{Positive Modulation}

Research on the importance of excitation in positive modulation has been aimed at assessing the extent to which modulation is merely an artifact of simple excitation. It is conceivable that greater responding on compound trials comes about because the feature's excitation summates with that of a weakly excitatory target. On the one hand, the early research focusing on the response topography suggested that a simple summation view would not provide a very satisfying account. However, we know little about how two excitatory CSs that elicit different conditioned responses (CRs) will summate in performance. As a result, the observation that responding on compound trials takes the form of responding to the target CS is not sufficient evidence that the feature modulates responding independently from its own excitation. 
The greatest potential for addressing this issue lies in research that has systematically examined the role that excitation plays in the ability of a stimulus to modulate. This research can be divided into two classes. One class of studies examined the modulatory capacity of a known excitor; another class of studies examined the effects of explicit changes in the modulator's excitation.

\section{Modulation by Excitors}

Rescorla (1985) described two autoshaping experiments that examined the ability of a simple excitor to modulate responding to a keylight target. In one experiment, either a $15-\mathrm{sec}$ white noise or a 15 -sec light was trained as a modulator signaling reinforcement of a 5 -sec keylight. The other diffuse stimulus was trained as an excitor by simply pairing it with food an equal number of times. Both diffuse stimuli were then tested for their ability to modulate responding to the keylight. As expected, responding to the keylight during the explicitly trained modulator was high. On the other hand, the diffuse stimulus trained as an excitor had little impact on responding. However, this result could also have resulted from greater familiarity with the modulator-target compound. The second experiment examined the modulatory ability of keylight stimuli that were trained as either modulators or excitors. Importantly, in this experiment, modulation was assessed by examining the ability of these keylights to augment responding to yet another keylight that had received simple excitatory training and extinction and had not otherwise participated as a target of modulation. Thus, neither compound presented during the test had been previously experienced by the pigeons. Although the excitor appeared to enhance responding to the target to some extent, responding was much greater when the target was signaled by the keylight that had been explicitly trained as a modulator. Rescorla (1987) has obtained similar results when the modulatory ability of a diffuse stimulus trained as either a modulator or an excitor was tested on a common transfer target. These experiments suggest that a stimulus trained as a modulator develops properties different from those of a simple excitor.

However, a less interesting but plausible possibility is that $\mathrm{AB}+, \mathrm{B}-$ training results in stronger excitation to $\mathrm{A}$ than does simple excitatory $\mathrm{A}+$ training alone. If so, the results above might merely reflect the modulator's greater excitatory strength. Associative models predict that A would acquire greater initial excitation during $\mathrm{A}+$ training than during $\mathrm{AB}+, \mathrm{B}-$ training; however, if training involves serial $\mathrm{A} \rightarrow \mathrm{B}+$ compounds where $\mathrm{A}$ terminates prior to the onset of $B$, as with Holland's preparation with rats or Rescorla's keylight modulators, A's excitation may be enhanced because B fills the "gap" between A and the US (see, e.g., Kaplan \& Hearst, 1982; Kehoe, Gibbs, Garcia, \& Gormezano, 1979; Pearce, Nicholas, \& Dickinson, 1981; Rescorla, 1982a).

In order to compare the excitatory strengths of diffuse stimuli trained as either positive modulators or excitors, Rescorla (1985; see also Holland, 1986a, 1986b) exam- ined the ability of each stimulus to act as a second-order reinforcer for a preceding keylight CS. Although both stimuli were effective in conditioning excitation to the new CSs, the stimulus originally trained as an excitor was a more effective reinforcer than was the stimulus trained as a modulator. Importantly, this result suggests that, under the present conditions, modulatory training results in weaker excitation than does simple excitatory training.

Although modulation appears to involve more than simple excitation, Brandon and Wagner (1991) have recently provided evidence that excitatory conditioning of a long-duration stimulus can provide that stimulus with the ability to enhance responding to another stimulus. Using rabbits in a conditioned eye-blink procedure, excitatory conditioning involved presenting a $50-\mathrm{msec}$ paraorbital shock US at varying times during a $30-\mathrm{sec}$ auditory stimulus. These temporal parameters were not sufficient for the auditory stimulus to elicit eye-blink conditioned responses (CRs); however, they found that CRs that were otherwise elicited by an excitatory shortduration CS were enhanced when that CS was tested in the presence of the longer excitatory stimulus. These results suggest that a long-duration excitatory stimulus can modulate responding to another excitatory $\mathrm{CS}$, when the two stimuli do not otherwise evoke similar CRs. Elaborating on a theory of associative acquisition proposed by Konorski (1967), Brandon and Wagner (see also Bombace, Brandon, \& Wagner, 1991; Brandon, Bombace, Falls, \& Wagner, 1991; Wagner \& Brandon, 1989) suggested that the long- and short-duration stimuli became associated with different aspects of the US. One feature of the excitation acquired by the long-duration stimulus was its ability to elicit preparatory CRs, whereas the shortduration CS elicited consummatory CRs. The preparatory CRs enhanced the magnitude of the consummatory CRs.

It is important to note that the ability of the longduration stimulus to enhance responding in these eyeblink experiments was acquired through simple pairings with the US. This modulatory function thus likely depends on the existence of an excitatory modulator-US association. If so, these findings are perhaps best understood in terms of the summation of excitation. Little is currently known about how excitatory stimuli that elicit different CRs will combine in performance, and these experiments provide some important insight into the mechanisms of excitatory summation.

On the other hand, as the research reviewed here suggests, there now exists a substantial body of evidence supporting the existence of a modulatory mechanism that involves more than simple excitatory associations. The term modulation as it is used here refers to situations in which one stimulus is used to influence responding to another stimulus in a manner that is orthogonal to its own associations with the US. The modulatory function demonstrated by Brandon and Wagner (1991) cannot easily be categorized in this manner. However, the conditions under which excitation-independent modulation is acquired are not well understood. With some proce- 
dures, it may be that CS-US pairings are sufficient for a stimulus to acquire both an excitatory US association and a modulatory function that is orthogonal to this association. Additional research will be required in order to address this possibility adequately.

In any case, the results of experiments comparing the modulatory and excitatory properties of stimuli suggest that positive modulation and excitatory training endow stimuli with separate properties. Although simple excitation may provide a stimulus with a functional ability to enhance responding to a target CS (and modulatory training can establish excitatory strength), Pavlovian positive modulation appears to involve more than simple excitation.

\section{The Effects of Changing the Modulator's Excitation}

A very successful technique for examining the role of excitation in modulation has been to manipulate the modulator's excitatory strength. Experiments from several preparations have examined the effects of conditioning excitation to the modulator both before and after modulation training, extinguishing excitatory responding to the modulator following modulation training, and either maintaining or suppressing excitatory responding to the modulator concurrently with training. The logic behind all of these manipulations is that the extent to which modulation depends on excitation should be revealed by the effect that a change in the modulator's excitation has on modulatory control.

Excitatory conditioning of the feature before modulation training. If modulation depends on a direct association between the feature and the US, one might expect that pretraining a feature-US association would enhance modulation. The effect of excitatory pretraining has been examined in both pigeon and rat preparations.

Using an autoshaping preparation with 15 -sec diffuse stimuli as modulators, Rescorla (1986b) conducted a systematic examination of the effects of pretraining excitation to either the A feature or the B keylight target on the subsequent acquisition of $\mathrm{AB}+, \mathrm{B}$ - discrimination learning. Far from enhancing modulation, the results showed that excitatory pretraining of A strongly disrupted differential responding to $B$. However, if $B$ had also received prior excitatory training, disruption was reduced. Rescorla suggested that excitation to A probably caused A to block excitatory conditioning of B. Excitatory pretraining of $B$ prevented complete blocking, thereby permitting control of responding by A. Because modulatory control was not enhanced by pretrained excitation but instead interfered with the development of responding to the target, these results provide further evidence that modulation is not simply a result of feature-US excitation.

In order to further assess whether excitation is actually incompatible with modulation, Rescorla (1986b) trained a compound of two diffuse stimuli as the A feature. One of the stimuli had received prior excitatory conditioning. If excitation and modulation are inherently incompatible processes, the excitatory element of the compound should become a weaker modulator during training. Although the extent of modulation of B was small-as was expected, given the previous results - both diffuse stimuli were similarly capable of enhancing responding to B when tested alone following training. These results suggest that excitatory training of the A feature probably disrupted modulation merely to the extent that it prevented $B$ from becoming excitatory.

The role of excitation has also been examined by using a serial $\mathrm{A} \rightarrow \mathrm{B}+, \mathrm{B}-$, appetitive preparation with rats. Ross (1983) examined the effect of excitatory pretraining of the visual A feature, the B target, and the $\mathrm{A} \rightarrow \mathrm{B}$ compound. He found that excitation to A slowed the acquisition of differential responding to B. Again, as with Rescorla's (1986b) findings, it is likely that the excitatory A blocked initial excitatory conditioning of B. In another group of rats, an excitatory B successfully blocked the development of A-US associations, but did not disrupt the ability of $A$ to subsequently modulate responding to $B$, which further suggests that $A$ need not be an excitor to modulate responding to $B$. Acquisition of the discrimination was enhanced, however, in a group that received reinforcement of the $\mathrm{A} \rightarrow \mathrm{B}$ compound prior to discrimination training. In summary, the results of Ross's (1983) experiments further support the contention that positive modulation is not due merely to excitatory training of the A feature alone.

Conditioning or extinction of the feature after modulation training. Several experiments have examined the relationship between excitation and modulation by manipulating the modulator's excitation following modulation training. These treatments have focused on the effect of reinforced or nonreinforced exposure to the $A$ feature alone following $\mathrm{AB}+, \mathrm{B}-$ discrimination training.

For example, using both localized keylights and diffuse stimuli as modulators in the autoshaping preparation, Rescorla has conducted several experiments in which modulators were differentially treated following training. The results demonstrated that neither posttraining reinforcement (Rescorla, 1985) nor extinction (Rescorla, 1986a) of the modulators had any effect on the modulatory strength of the stimuli.

The results from studies using the appetitive observational preparation with rats, however, are somewhat mixed. Ross (1983) found considerable disruption of modulation after the $A$ feature had been presented alone following training, although modulation was rapidly reacquired during continued training. Presumably, exposure decreased attention to the feature, which would have the effect of making the reinforced and nonreinforced target presentations less discriminable. On the other hand, Holland $(1989 \mathrm{~b}, 1989 \mathrm{c})$ has typically found that extinction of A produces little disruption of modulation. In fact, Holland (1989b) found that presentations of $A$ following $A \rightarrow B+, B-$ training actually enhanced the ability of $A$ to control responding to a different target from another modulatory discrimination. In agreement with the results of Rescorla (1985), Ross (1983) found that reinforcement of A following training had no effect on A's modulatory strength. 
In general, modulation does not appear to be strongly affected by either nonreinforced or reinforced exposure to the modulator following discrimination training. These results again support the view that excitation is not an integral component of modulation.

Changes in the feature's excitation during training. A further technique for assessing the role of excitation in positive modulation is to examine the effect of modulator-US manipulations that take place concurrently with discrimination training. Research has mainly focused on nonreinforced separate presentations of the modulator.

Ross and Holland (1982) examined modulation with a serial positive patterning, $\mathrm{A} \rightarrow \mathrm{B}+, \mathrm{B}-, \mathrm{A}-$, procedure. As with the serial feature-positive procedure, a tone, $B$, was reinforced with food only when it followed a light, A. However, both the tone and light were nonreinforced when presented alone. Although this experiment did not include a control group for which the light was not separately presented, differential responding to the tone on $\mathrm{A} \rightarrow \mathrm{B}+$ and $\mathrm{B}-$ trials was comparable to that seen in previous research (e.g., Ross \& Holland, 1981). Further, direct associations between the modulatory light and the US appeared to be weakened as a result of separate light presentations. This result provided an initial indication that concurrent presentations of the modulator alone do not disrupt modulation.

Subsequently, Holland and Block (1983) examined positive patterning with a 10 -sec simultaneous, as opposed to serial, compound of the light and tone. Ross and Holland (1981) had suggested previously that if A and B are presented simultaneously during $\mathrm{AB}+, \mathrm{B}-$ training, $A$ becomes a simple excitor and does not modulate responding to B. However, this simple excitatory control by either the light or the tone should be hindered by the separate nonreinforced presentation of both stimuli in the patterning procedure. They noted the development of a "rear/headjerk" response on reinforced compound trials and suggested that this otherwise infrequent response may be evidence that a cue unique to the light-tone compound was acquiring excitation (e.g., Rescorla, 1973). Note, however, that the use of a new mutually exclusive class of responding that is a subset of both rear and headjerk must necessarily reduce the number of scored occurrences of these independent behaviors. In fact, when categorized in the same manner as in prior research, headjerk on light-tone compound trials appeared markedly greater than to the tone alone. This pattern of responding has often been used to support the postulation of a modulatory mechanism. As will be discussed later, Holland (1989a) has provided stronger evidence favoring the use of a configural mechanism during positive patterning training.

Recently, Holland (1989b) has shown that presentations of $\mathrm{A}$ alone during serial training both decrease A-US associations and enhance A's ability to modulate responding to a transfer target that has been trained in either another patterning or a traditional feature-positive discrimination. Because excitation was reduced, these results suggest further that positive modulation does not depend on excitation.

\section{Negative Modulation}

As with positive modulation, much of the negative modulation research that has examined the role of simple associations has focused on the effect of excitation on modulation. In fact, convincing evidence for a negative, or inhibitory, modulatory role in Pavlovian conditioning comes largely from the demonstration that certain training procedures establish inhibitory control that appears to be independent of direct associations with the US. Research has examined both the extent to which negative modulators can have concurrent excitatory associations and the impact of excitatory training on inhibitory control.

Several authors have examined various techniques for assessing the independence of inhibitory control from simple associations with the US. Both Rescorla (1985) and Jenkins (1985) have used the autoshaping preparation to examine the effect of excitatory training of the modulator on modulatory control. Rescorla (1985) trained pigeons on two feature negative, $\mathrm{AB}-, \mathrm{B}+$, discriminations in which the inhibitors, $A$, were colored keylights followed immediately by either a white key or an X pattern, B. Presentations of B were otherwise reinforced. When the inhibitors were compared to a stimulus that had been nonreinforced alone during training, control acquired by each inhibitor transferred well to the excitor that had been trained with the other inhibitor. Then, in order to establish differential levels of excitation to the inhibitors, one inhibitor was reinforced and the other was presented alone. A second test of inhibition revealed that both inhibitors still suppressed responding to the excitors, although the inhibitory strength was reduced for the stimulus that had received excitatory training. However, when both inhibitors were then repeatedly nonreinforced, inhibition was again strong and equivalent for both inhibitors. This suggests that the disruption of inhibition that followed excitatory training was possibly due to a masking of the inhibitory control by excitatory responding (see also Hearst, 1984). When excitatory responding was reduced, inhibitory control reappeared.

Jenkins (1985) also examined the effect of excitatory training of an inhibitor in an autoshaping preparation. He used a simultaneous Pavlovian conditioned inhibition preparation, in which a target keylight was paired with the US except when it was presented in compound with a 6-sec white noise stimulus. On reinforced trials, the grain US occurred at the end of the 6-sec target, or following four pecks at the target. Following successful acquisition of the discrimination, one group of subjects received noise-US pairings; another group received the noise-keylight compound paired with the US; and a control group received no further treatment with the noise. Consistent with Rescorla's (1985) results, subsequent tests of inhibitory control revealed that excitatory training of the noise alone had no detrimental impact on in- 
hibition. Furthermore, the noise continued to exert inhibitory control over the keylight when the noise-US trials were subsequently intermixed with inhibitory training.

Holland (1984; see also Holland, 1989d; Morell \& Holland, 1993) has also examined counterconditioning of inhibitors, specifically looking at differences resulting from simultaneous, or $\mathrm{AB}-, \mathrm{B}+$, and serial, $\mathrm{A} \rightarrow \mathrm{B}-, \mathrm{B}+$, conditioning procedures. Holland and his collaborators (e.g., Holland, 1985; Holland \& Gory, 1986; Holland \& Lamarre, 1984) have suggested that serial inhibitory training produces negative modulation that should not be affected by the concurrent presence of excitation. Excitatory conditioning of simultaneously trained inhibitors, on the other hand, should be retarded (see, e.g., Rescorla, 1969) and should disrupt previously established inhibitory control. Using the conditioned suppression preparation with rat subjects, Holland (1984) established inhibition by training either serial or simultaneous feature-negative discriminations in which a $60-\mathrm{sec}$ excitatory CS, B, was paired with a shock US when presented alone, but was nonreinforced when preceded by (in one group), or presented simultaneously with (in another group), a $60-\mathrm{sec}$ feature, A. After responding to B came under the control of $A$, animals received pairings of $A$ with shock until A elicited a strong excitatory CR. Consistent with Holland's predictions, the group trained with a serial feature-negative procedure showed fast acquisition of excitation when compared with the group given simultaneous training. More importantly, subsequent testing of inhibitory control revealed that the excitatory training had little effect on the ability of the serially trained feature to inhibit responding to the excitor, whereas inhibitory control by the simultaneously trained feature was considerably undermined.

\section{Alternative Roles for Excitation}

In general, observations that excitatory training does not destroy the negative modulatory ability are among the strongest evidence that modulation does not function in a manner consistent with simple associative models. However, under some circumstances, excitation may play an important role in modulation. Recently, Rescorla (1991b; see also Robbins, 1990) has reported that concurrent excitatory training can actually enhance the development of modulation. In several experiments with pigeons in the autoshaping preparation, the effect of concurrent reinforced or nonreinforced presentations of the feature on positive and negative modulation was examined. The results showed that regardless of the type of modulator, separate reinforcement improved its modulatory ability. Because current associative theories predict that excitation should reverse the effects of inhibitory feature-US associations, these results provide especially powerful evidence for the existence of an inhibitory modulatory mechanism that is separate from direct US associations. Rescorla suggested that rather than affecting the US representation directly, excitation may improve modulation by increasing the salience and/or processing of the feature.
In combination with the results of experiments demonstrating that modulation does not involve a simple associative mechanism, Rescorla's (1991b) observation that excitatory training can sometimes enhance both positive and negative modulatory control suggests that excitation may play a more elaborate role in modulation. This finding highlights the importance of controlling for excitation in investigations of modulation. However, the extent of excitation acquired during training is often confounded with the procedures used to establish modulation. For example, although Holland (e.g., 1984) has suggested that simultaneous and serial feature-negative procedures establish inhibitors that use different learning mechanisms, it is important to note that the two procedures also establish markedly different magnitudes of secondorder excitation as well (see, e.g., Holland, 1984; Holland \& Lamarre, 1984; Rescorla, 1982b). The possibility that excitation or excitatory CRs may play a role in inhibitory learning may account for some of the differences found with these two training procedures. The importance of this distinction will be discussed in more detail below.

Instead of attempting to control for the level of excitatory conditioning involved in feature-positive training, investigators in several laboratories have often used the tactic of controlling for the procedural relationship of the modulator with both the target and the US. For example, in several experiments, the modulatory ability of the putative modulator $\mathrm{A}$, trained in an $\mathrm{AB}+, \mathrm{B}$ - procedure has been compared with that acquired in a "pseudofacilitation" $\mathrm{AB}+, \mathrm{B}+$ procedure (see, e.g., Davidson, Aparicio, \& Rescorla, 1988; Davidson \& Rescorla, 1986; Rescorla, 1985; see also Holland, 1986b; Wilson $\&$ Pearce, 1989). The advantage of this procedure is that although the modulator and pseudofacilitator are paired an equal number of times with $B$ and with the US, the pseudofacilitator provides no information about B's reinforcement. Results have consistently shown that the pseudofacilitator has little or no modulatory strength. This procedure does not control perfectly for simple excitatory strength acquired by $\mathrm{A}$; the pseudofacilitator may acquire more second-order excitation, whereas associative models would predict that the pseudofacilitator would acquire less excitatory strength. However, until more is known about the extent and nature of the excitation acquired in feature-positive procedures, controlling for informational strength may be the most appropriate strategy.

\section{Summary}

In summary, the relationship between modulation and simple associative processes appears complex. There is sufficient evidence to suggest that both positive and negative modulation involve processes that are distinct from simple excitation. On the other hand, under some circumstances, excitors can modulate, and modulators become excitatory. However, the degree to which stimuli trained as simple excitors can modulate responding through a mechanism that is distinct from a simple associative mechanism requires further investigation. Finally, exci- 
tation has now been shown to augment both the acquisition and expression of modulation. Although the excitatory and modulatory processes can be independent, the complete role that excitation might play in modulation is still unclear.

\section{EXAMINING THE MODULATORY MECHANISM}

After having reviewed the evidence on the role of simple excitation in modulation, we have concluded that modulation involves a mechanism that is relatively independent of simple US associations. If not through direct associations with the US, how might modulators affect responding to the target? The alternative mechanisms that have been suggested differ with respect to the locus of the modulatory action. Rescorla and Holland (1977), for example, in their investigation of inhibitory control, suggested that there are four potential loci of inhibitory action. Inhibitors may act by directly affecting the representations of the target CS, the CR, the CS-US association, or the US. More recently, Rescorla (e.g., 1985) and Holland (e.g., 1985) have emphasized these loci for the potential action of both positive and negative Pavlovian modulators.

First, there are several mechanisms through which a modulator might act directly on a specific CS. One possible CS-specific mechanism that has received considerable discussion in the literature is the possibility that the modulator configures with the target on compound trials so that the feature-target compound is perceived as a unique stimulus. Solution of the discrimination would thus involve a simple Pavlovian discrimination between two distinct events.

Second, instead of acting directly on the target CS, a modulator might act on the CR evoked by the CS. Positive modulators might acquire the ability to enhance a particular response, whereas negative modulators might interfere with or suppress the evocation of a specific response.

A third possibility is that modulators act directly on established CS-US associations. Holland (e.g., 1983) has proposed that modulators act in a hierarchical manner to control the expression of the specific CS-US association involved in training the modulator. Positive modulators enable or activate the association, whereas negative modulators suppress or inhibit the association. More recently, Holland (1989d) has suggested that a modulator's action may not be restricted to a specific association, but instead may be more general to a particular class of CS-US associations-namely, associations that have come under previous modulatory control.

The fourth possible locus is the US itself. Rescorla (e.g., 1985) has suggested that modulators act on the US so as to change the threshold for activation of the US representation. Within this view, modulators change the likelihood that a target will activate the US representation, thereby evoking a response. Positive modulators lower the US threshold, which would facilitate the ability of such a target to activate the US. A negative modulator acts by raising the US threshold, thus suppressing the expression of an excitatory CS-US relation. This interpretation of negative modulation is similar to one proposed by Konorski (1948; see also Rescorla, 1979) as an explanation for the action of conditioned inhibitors.

\section{Transfer Assessments}

One extensively used technique for assessing the nature of modulation has been the investigation of the modulator's ability to control responding to target stimuli other than the original training target. If modulators act directly on either the CS or a specific CS-US association, then a modulator that has been trained with one CS should have little impact on responding to a CS that has not been trained with that modulator. Similarly, if the modulatory impact is on the CR, modulators should not control responding to CSs that elicit different CRs. On the other hand, if modulation involves changes in some attribute of the US representation (e.g., the threshold for its activation), then all targets that are sensitive to changes in that attribute should be affected by the presence of the modulator.

Experiments involving transfer have focused on a number of different issues. The specificity of modulation to the original CS has been examined in several preparations and has received considerable attention because of its potential for exposing the nature of modulatory control. Transfer has also been used simply as a tool for assessing the effectiveness of a modulator; it is often desirable to compare the modulatory abilities of two or more stimuli on a common target that has not received prior training with the putative modulators. In these situations, differential transfer is usually taken as evidence of differential modulatory strength.

Unfortunately, many experiments that have assessed transfer of modulation across targets have failed to compare the modulatory control with that of a stimulus that does not receive explicit modulatory training. Without such a comparison, it is impossible to assess the extent to which the modulator's effect on responding to the transfer target is attributable to the conditioned as opposed to the nonassociative properties of the modulator. Although this does not represent a major concern in experiments that compare the modulatory strength of differentially treated modulators, the CS specificity of modulation cannot be determined without accounting for the nonassociative effects of the modulator.

\section{Positive Modulation}

Rescorla and his collaborators have examined the CS specificity of positive modulation in a number of situations, using autoshaping with pigeons. To summarize, positive modulators consistently enhance responding to transfer targets that have been involved in other positive modulation discriminations (e.g., Rescorla, 1985; Rescorla, 1986b), although the extent of modulation is often weaker than that observed in the original modulatortarget compounds. Further, modulators will enhance responding to a stimulus that has been trained and extin- 
guished (e.g., Rescorla, 1985, 1987, 1988, 1991b, 1991c; Swartzentruber \& Rescorla, 1994), and such enhancement is greater than that demonstrated by a stimulus that has not received modulatory training (e.g., Rescorla, 1985; Swartzentruber \& Rescorla, 1994). Similarly, in comparison with a stimulus that has received simple excitatory training, a positive modulator will enhance responding to a compound consisting of excitatory and inhibitory elements (Rescorla, 1987). On the other hand, positive modulators do not enhance responding to stimuli that have received nonreinforced preexposure, simple excitatory training, or excitatory conditioning with a trace procedure (e.g., Rescorla, 1985). In these experiments, however, the effect of the modulator was not compared with the effect of a control stimulus lacking modulatory training.

Recently, Swartzentruber and Rescorla (1994) used the autoshaping preparation to compare the extent to which positive and negative modulation transfers to a stimulus that has been trained and partially extinguished and to a stimulus that has received nonreinforced exposure prior to partial excitatory training. In an attempt to rule out the effect of any unconditioned properties of the modulators on transfer, we compared transfer by the modulators to the effect of pseudomodulatory control stimuli that had not received explicit modulatory training. Furthermore, in order to compare the sensitivity to transfer of stimuli with different training histories, it was important to examine transfer at a point when responding to the separate stimuli was equivalent. Thus, after modulation and pseudomodulation training, pigeons received discrimination training with two stimuli, $C$ and $D$, in which $C$ was reinforced and $D$ was nonreinforced. Transfer by the modulator and pseudomodulator was then assessed while $C$ and $D$ were undergoing a discrimination reversal. Thus, $\mathrm{C}$ was undergoing extinction, whereas $\mathrm{D}$ was undergoing initial excitatory acquisition. Results demonstrated that transfer of both positive and negative modulation was reliably greater to the extinguishing stimulus, $C$, than to the acquiring stimulus, $D$, suggesting that an extinguishing stimulus is more sensitive to modulatory control than a stimulus that has received nonreinforced exposure prior to conditioning. Because the identity of C and D was counterbalanced, this design ruled out the possibility that transfer results solely from stimulus generalization between the perceptual features of the original training target and the transfer targets.

A number of experiments have examined transfer of positive modulation in appetitive preparations with rats. The ability of a modulator to influence responding to different targets also depends importantly on the training history of these targets, but results are not completely consistent with those from the autoshaping preparation. Modulators will enhance responding to targets that have been trained in similar positive modulation discriminations (Davidson \& Rescorla, 1986; Holland, 1986a, 1989a; Wilson \& Pearce, 1989, 1990; but see Holland, 1986b), and such transfer is strongest if transfer is tested following nonreinforced exposure to the modulator, or if training involves a patterning procedure where the modulator is nonreinforced alone on some trials during training (e.g., Holland, 1989b). Positive modulators do not enhance responding to partially reinforced stimuli (Holland, 1983, 1986b; Wilson \& Pearce, 1990) or to a target consisting of a compound of both excitatory and inhibitory elements (Holland, 1986b). Experiments testing transfer to trained and extinguished stimuli have provided mixed results. Holland (e.g., 1986a, 1986b, 1989a, $1989 \mathrm{~b}$ ) has typically found little or no enhancement, whereas other researchers have observed enhancement (e.g., Jarrard \& Davidson, 1991; Davidson \& Jarrard, 1989). The reason for this discrepancy is unclear; however, Holland (1991a) has recently observed enhancement of responding to an extinguished excitor when modulation was trained in a discrete-trial operant procedure with rats.

Although there are a few inconsistencies across laboratories and preparations, researchers generally agree that positive modulators can transfer control across targets. Evidence for transfer suggests that positive modulators act, at least in part, in a manner that is independent of the identity of the original CS. The extent of transfer, however, depends on the nature of the transfer target's prior training; targets with currently suppressed levels of excitation and targets of other positive modulation discriminations appear most sensitive to positive modulatory control.

\section{Negative Modulation}

The ability of negative modulators to influence responding to transfer targets has also received considerable investigation. The research in this area has focused on the extent to which serial, $\mathrm{A} \rightarrow \mathrm{B}-, \mathrm{B}+$, and simultaneous, $\mathrm{AB}-, \mathrm{B}+$, training procedures differentially affect responding to transfer targets, and on the extent to which the conditioning history of the transfer target determines the extent of modulatory transfer.

Using conditioned suppression with rats, Holland and Lamarre (1984) examined the abilities of serial and simultaneous inhibitors to transfer to a simple excitatory CS. Importantly, throughout testing, inhibition in both groups was compared with that seen by a control group that had received $\mathrm{A}-, \mathrm{B}+$ discrimination training, which might itself have produced differential inhibition. When testing was conducted with the same temporal parameters that were used in training, the results revealed reliable transfer only by the simultaneous group. Serial inhibitors showed no evidence of transfer to a simple excitor. Note, however, that this test was conducted under different temporal conditions for the different groups. When Holland and Lamarre tested the simultaneously trained animals serially and the serially trained animals simultaneously, neither group showed transfer to the excitor. In fact, with serial testing, the simultaneous group no longer suppressed responding to its original excitor.

In a second experiment, Holland and Lamarre (1984) used a within-subjects design to examine the extent to which serial and simultaneous inhibitors suppress re- 
sponding to the target from the other discrimination. The serial inhibitor transferred weakly to the excitor from the simultaneous discrimination when testing was either serial or simultaneous, and there was good inhibition of the serial inhibitor's own excitor, regardless of testing procedures. On the other hand, the simultaneous inhibitor suppressed responding to the serially trained excitor when tested both serially and simultaneously but again did not inhibit its own excitor when tested serially. As suggested by Holland and Lamarre, the observation that the simultaneous feature showed better control of the transfer excitor than of its original excitor during serial tests may have been due to generalization across the two inhibitors during serial testing. Alternatively, this result might suggest that responding evoked by a serially trained excitor is more sensitive to inhibitory control, or unconditioned disruptive effects, than is either an excitor from a simultaneous discrimination or a CS that has received simple excitatory training. Unfortunately, because suppression was not compared with that induced by a control stimulus, it was not possible to assess the extent to which the observed transfer resulted from inhibitory training.

In a series of experiments, using conditioned suppression, Lamarre and Holland (1987) examined the extent of transfer by serially trained negative modulators across a range of different targets. During serial tests, they found strong transfer to a target trained in another serial feature-negative discrimination, weaker transfer to a target from a simultaneous discrimination, and little or no transfer to a simple excitor, a partially reinforced excitor, and a stimulus that had undergone conditioning, extinction, and reconditioning.

Holland (1989d) recently examined transfer of negative modulation, using an appetitive observational preparation with rats. The results of several experiments revealed that serially trained inhibitors show stronger transfer to targets from other serial discriminations than to targets that have received simple excitatory training (see also Holland \& Coldwell, 1993, and Morell \& Holland, 1993, for additional support from a discrete-trial operant procedure with rats). Furthermore, retraining a serial inhibitor's target with a different appetitive US disrupts inhibitory control. Control by simultaneously trained inhibitors, on the other hand, is less affected by this manipulation. Interestingly, Holland found that serial inhibitors did transfer across USs if the subjects had originally received concurrent training with a second serial feature-negative discrimination involving this new US. Holland (1989d) proposed that modulatory training of the targets and USs placed them in a "higher order memory system." He suggested that transfer across targets and USs may occur only when both the target and the US on which its excitation is based are represented in this system.

In general, the results of these experiments suggest that whereas simultaneous features appear capable of suppressing responding to a range of excitors (but only when tested simultaneously), the extent to which a seri- ally trained feature will influence responding depends on the conditioning history of the target. The strongest control is typically seen when serially trained features are tested on targets from other serial discriminations. The occasionally observed transfer to other targets may be due to generalization or unconditioned disruption.

The importance of the target's training history to inhibitory transfer is complicated by the difficulty in making direct comparisons of serial and simultaneous inhibitors under identical testing conditions. Although simultaneously trained inhibitors often show quite strong transfer across different targets, most experiments have provided much weaker or no evidence of transfer by simultaneous inhibitors when testing occurs serially (Detke, 1991; Holland, 1984, 1989d; Holland \& Lamarre, 1984; Rescorla, 1989; but see Holland \& Gory, 1986). Furthermore, although transfer by serially trained features is seen when testing occurs either serially or simultaneously, it is often confined to targets from serial discriminations (see, e.g., Lamarre \& Holland, 1987; but see Rescorla, 1985, 1989).

Several researchers have used the autoshaping preparation with pigeons to examine transfer of negative modulation to targets with various conditioning histories. Negative modulators have been found to suppress responding to targets that have received simple excitatory training (Rescorla, 1989, 1991b); excitatory training and partial extinction (e.g., Swartzentruber \& Rescorla, 1994, described earlier); or training, extinction, and retraining (e.g., Rescorla, 1987, 1991a, 1991c). As with positive modulation, negative modulators typically show strong transfer to targets from other discriminations (e.g., Rescorla, 1985). And although Jenkins (1985) found little initial transfer of inhibitory modulation to a simple excitatory keylight target, inhibition to this target was acquired very rapidly during subsequent inhibitory training.

It is difficult to summarize the results of the inhibitory transfer experiments. As with positive modulation, the results from experiments with rats, conducted, for example, by Holland and his collaborators, have demonstrated that the extent of transfer appears to depend on both the inhibitory training procedures and the training history of the transfer target (see also Wilson \& Pearce, 1989, 1990, described in more detail in the next section). Examinations of inhibitory control with pigeons, on the other hand, have found that transfer depends less on the specific temporal characteristics of the training and testing procedures, but is influenced by the training history of the transfer target.

\section{Generalization}

Although observed transfer appears to suggest that modulatory action is not directed at a specific CS or CS-US association, much of the evidence for transfer could be explained through stimulus generalization resulting from the perceptual similarity of the original and transfer targets. If the targets have each been trained in similar discriminations, generalization could also occur between their separate features. One way in which these 
possibilities have been effectively addressed can be found in studies that examine the extent to which modulation will transfer across targets that differ in modality. The assumption underlying this strategy is that generalization is unlikely across such targets. Both rat (Davidson \& Rescorla, 1986; Holland, 1989a; Lamarre \& Holland, 1987) and pigeon (Rescorla, 1985) preparations have provided evidence that modulators trained with either visual or auditory targets will also control responding to an appropriately trained target from the other modality. These results suggest that transfer is probably not based entirely on the perceptual similarity of the targets.

Recently, Pearce and his collaborators have suggested that generalization may play a broader role in learning than has traditionally been considered. Pearce (1987) proposed a model of learning in which the entire stimulus complex present on a given trial is processed as a single configured stimulus. Excitatory conditioning consists of the formation of an association between this compound stimulus and the US. Unlike traditional views of compound conditioning, in which each element acquires an independent excitatory association, Pearce suggests that only one association is formed. The extent to which each element of the compound will subsequently evoke a CR reflects generalization between the excitatory compound and the element. In feature-positive learning, $\mathrm{AB}+\mathrm{B}-$, excitation conditioned to the $\mathrm{AB}$ compound initially generalizes to $\mathrm{B}$ alone. As a result of B's nonreinforcement, B eventually acquires inhibitory strength that offsets the generalized excitation. Similarly, feature-negative learning, $\mathrm{AB}-, \mathrm{B}+$, results in an excitatory $B$ stimulus and an $A B$ compound with inhibitory strength sufficient to offset generalized excitation from B. Thus, in general, feature-positive and -negative discriminations result in the learning of simple discriminations between two similar but unique events.

In order to compare the separate views of modulatory learning with the predictions of the Pearce (1987) model, Wilson and Pearce $(1989,1990)$ have examined discrimination learning, using an appetitive observational preparation with rats. In their procedure, a 2-min feature stimulus signaled whether or not embedded presentations of a 10-sec target CS would be followed by food. For example, in one experiment (Wilson \& Pearce, 1989), a target $\mathrm{CS}, \mathrm{B}$, was reinforced when presented during $\mathrm{A}$, but nonreinforced during C. According to Pearce (1987), such training would result in the $\mathrm{AB}$ compound acquiring excitatory strength, whereas the CB compound would become inhibitory. As the discrimination was learned, the animals showed more frequent entries into the food magazine during the $A B$ compound than during $C B$. In the second phase, in addition to the $\mathrm{AB}+, \mathrm{CB}-$ discrimination, the animals were trained on a second, $\mathrm{AD}-, \mathrm{CD}+$, discrimination. Note that this discrimination precluded a simple solution in terms of A's positive excitatory or modulatory strength and/or C's negative strength. Nevertheless, the results showed little disruption of the original discrimination as the second discrimination was acquired. Solution of such a conditional discrimination can only occur through a mechanism that involves learning about specific compounds of stimuli.

However, in a second experiment, Wilson and Pearce (1989) found that a feature from one feature-positive discrimination reliably enhanced responding to a target from a second feature-positive discrimination. Although other researchers had taken such transfer as evidence that the features modulate responding independently of CS identity, Wilson and Pearce noted that transfer is consistent with the possibility that subjects generalized across the reinforced compounds experienced in training. Thus, they suggested that transfer did not necessarily reflect the existence of independent modulatory control.

In a subsequent series of experiments, Wilson and Pearce (1990) investigated the generality of their transfer results. Feature stimuli from both feature-positive and feature-negative discriminations exerted strong control over responding to targets from other similar discriminations, but little or no transfer to CSs that had received simple excitatory training or partial reinforcement. Pearce's generalization account does not predict differential transfer to targets that otherwise evoke similar levels of responding. However, the results are consistent with those of Lamarre and Holland (1987) and Rescorla (e.g., 1985) in showing that the extent of transfer depended on the training history of the target.

Pearce and Wilson (1991) have recently conducted another series of experiments in which they examined the role that generalization plays in discrimination learning. Using appetitive preparations with rats and pigeons, they provided evidence consistent with Holland (1984) and Rescorla (1985), that excitatory conditioning of a conditioned inhibitor does not always abolish inhibitory control. Inhibition was still revealed during simultaneous compounds of the inhibitor and excitor, providing a powerful demonstration that inhibition was preserved.

Although Holland and Rescorla had taken their results as evidence of inhibitory modulation, Pearce's (1987) model of stimulus generalization provides an alternative account of this effect. As an animal learns an $\mathrm{AB}-, \mathrm{B}+$ discrimination, $B$ enters into an excitatory association with the US, whereas the $\mathrm{AB}$ compound acquires inhibitory strength to offset the generalized excitation from B. During subsequent conditioning of $\mathrm{A}, \mathrm{A}$ acquires an excitatory association of its own. However, when the inhibitory $\mathrm{AB}$ compound is presented again during the final test phase, the extent to which $A B$ now evokes excitatory responding depends only on AB's similarity to $A$ and $B$ alone. The prediction is thus that A's excitation will only partially mask AB's conditioned inhibition.

An explanation of this pattern of results from a modulation perspective suggests that, during simultaneous tests, A's influence over B must transcend its own response evocability. The analysis offered by Pearce and his collaborators does not require this assumption. On the other hand, according to Pearce's model, inhibitory strength is possessed by the AB compound. Neither A nor $B$ is itself inhibitory. As a result, the model inherently predicts that inhibitory control by $\mathrm{A}$ is specific to 
B. Excitatory conditioning of A should abolish A's ability to inhibit targets other than B. Although there are procedural differences, Rescorla (1991b) has recently found that reinforced, compared with nonreinforced, presentations of an inhibitor during training enhanced transfer of inhibition. On the other hand, two experiments provide data on the effect of post training excitatory conditioning on transfer of inhibition. Holland (1989d), using the appetitive observational preparation with rats, found that transfer of a simultaneously trained inhibitor's control was abolished by excitatory conditioning. However, Holland (1991a), using a discrete-trial instrumental procedure with rats (discussed in more detail later), found that excitatory training of a serially trained inhibitor had little effect on inhibitory control of the original excitor, but that transfer to a different target was disrupted. The latter results are especially consistent with the Pearce model, which predicts that $\mathrm{AB}-, \mathrm{B}+, \mathrm{A}+$ training would establish especially strong inhibition to the $\mathrm{AB}$ compound, but that $\mathrm{A}$ itself would be excitatory and therefore would not inhibit responding to an excitatory transfer CS.

One potentially fruitful elaboration on Pearce's (1987) model that has received some empirical support in the literature is to assume that generalization between stimuli is enhanced between stimuli with similar training histories. Honey and Hall (1989), for example, found that rats will be more likely to show more generalization of conditioned suppression between two stimuli that have had similar appetitive conditioning histories than between two stimuli that do not share a similar history. The existence of such an acquired equivalence mechanism can go a long way toward explaining much of the transfer data in terms that are consistent with a CS-specific account of modulation. For example, although modulators might act on specific CS-US relations, as originally hypothesized by Holland (1983), transfer would be predicted to occur to targets that share similar histories with the training $\mathrm{CS}$, such as those from other modulation discriminations. In fact, as mentioned previously, such transfer is typically quite strong regardless of the modulatory preparation.

In summary, although no single account of modulation has proved successful in predicting the entire pattern of transfer results, the model proposed by Pearce (1987) provides important insight into the role of generalization in learning. It can account in large part, but not completely, for learning of the discriminations, independence of modulation from direct associations with the US, and transfer test performance.

\section{Action on the CR}

The possibility that positive and negative modulators act on a specific CR has received relatively little direct attention. Most examples of transfer across target CSs use targets that elicit the same CR. However, because stimulus modality has been found to be an important determinant of the response (see, e.g., Holland, 1977), the account can be addressed with data from the few studies that examine transfer to stimuli from different modali- ties. In a feature-positive experiment reported by Rescorla (1985), for example, separate keylights were trained as positive modulators for either another keylight or an auditory target, each of which was otherwise nonreinforced. Recall that excitatory keylights elicit keypecking, whereas excitatory diffuse auditory stimuli elicit an increase in general activity that is not expressed as directed pecking. An additional keylight, employed as a control for modulation, was trained in the same relation with the US as were the modulators, but it did not signal reinforcement of a target CS. Transfer was then assessed on yet another trained and extinguished keylight. The results showed that regardless of the modality of the original training target, both features greatly enhanced pecking at the transfer keylight. Importantly, although the control keylight trained in a trace relation with the US also elevated responding to the target somewhat, this elevation was much weaker, suggesting that explicit modulatory training was necessary for transfer. These results suggest that the modulatory action is not specific to either the original target or its CR. Holland (1989a, described in detail in the next section) has provided data consistent with this conclusion using the observational preparation with rats.

Two experiments by Davidson et al. (1988), who used rat subjects, provide further evidence against a specific response as the locus of modulatory action. They investigated the interchangeability of Pavlovian modulators and stimuli trained as instrumental discriminative stimuli $\left(\mathrm{S}^{\mathrm{D}} \mathrm{s}\right)$. The results showed that a positive modulator was capable of enhancing the rate of a barpressing response that had come under discriminative control, and that an $\mathrm{S}^{\mathrm{D}}$ reliably facilitated headjerk responding to the target of a Pavlovian modulator. Pseudomodulators were used as controls in both experiments. As mentioned above, such stimuli might not control adequately for the excitatory associative strength acquired by the modulator and $\mathrm{S}^{\mathrm{D}}$. However, it is worth noting that because the target responses that are controlled in Pavlovian and instrumental paradigms differ markedly, the results of these transfer experiments provide additional evidence against the possibility that modulators act on a specific response.

\section{Is Modulation CS Specific?}

In spite of some mixed results from transfer experiments conducted in different laboratories and with different procedures, most investigators have taken the transfer data as sufficient evidence that the locus of modulation is not simply the CS alone or the CS-US association. However, the ability of a modulator to affect responding to transfer CSs is seldom as strong as its control over responding to the target used in training (e.g., Morell \& Holland, 1993), suggesting that some degree of the modulatory action is due to an attribute of the original modulator-target compound. Several possible explanations have been proposed to account for incomplete transfer. These include the contribution of the hierarchical mechanism suggested by Holland (e.g., 1983,1985 ), where modulatory action is on a specific 
CS-US association, and direct action on the CS, perhaps through a configural process. The contribution of either of these processes would reduce the extent of transfer across different targets.

The possibility that the modulator and target stimulus form a configured stimulus during training has been discussed by several researchers. Most arguments, however, are directed against the likelihood that configural cues form the sole basis of modulation. No one argument is adequate to discount configuring as at least a partial contributor to modulation.

However, Ross and Holland (1981) and Holland (e.g., 1983, 1986a; see also Arnold, Grahame, \& Miller, 1991; Holland \& Lamarre, 1984) have argued that an $A \rightarrow B+, B-$ procedure in which $A$ and $B$ are temporally separated should make the configuring of A and B less likely. Furthermore, a configural solution of a feature-positive discrimination should become more difficult as the interval between $A$ and $B$ increases. In fact, using a visual A stimulus and an auditory $B$, these authors have shown that extending the $\mathrm{A} \rightarrow \mathrm{B}$ interval up to a point produces greater differential headjerking to $B$, suggesting enhanced modulation. However, because we do not yet have an adequate understanding about the interactions between excitatory visual and auditory stimuli, it is often difficult to compare the response magnitude and rate of discrimination learning across procedures that use different temporal intervals. For example, although it is unclear what response would be elicited by serial and simultaneous visualauditory configures (but see Holland \& Block, 1983, for a possible "rear/headjerk" candidate), it seems plausible that the insertion of an empty trace interval between the two stimuli could affect the form of this response without changing the nature of the learning mechanism.

In an effort to assess the role of stimulus configuring in modulation, Holland (1989a) has compared the acquisition rates and extent of modulatory transfer when the A feature and B target were from either the same or a different modality. The major assumption underlying this strategy was that any role for configural processes in discrimination learning would be enhanced by the use of same-modality stimuli. The designs of the experiments were elaborate. In addition to manipulating modality, Holland examined the effect that presentations of $\mathrm{A}$ alone (patterning) would have on either serial (Experiment 1) or simultaneous (Experiment 2) discrimination learning and transfer. In order to test transfer, rats from each of the eight conditions were given concurrent training on two separate discriminations. Transfer across similarly trained targets would be more likely if a modulatory mechanism was being used than if the discriminations involved configural learning.

This design made several assumptions about occasion setting, configuring, and the involvement of unique cues, which are stimuli hypothesized to exist only during the compound presentation of other stimuli (see Rescorla, 1973). First, stimuli from the same modality should be more readily configured than stimuli from different modalities. Second, modulatory learning should be favored by a serial presentation of the stimuli on reinforced compound trials, whereas configural learning would be encouraged by simultaneous training. Thus, transfer should be more likely following serial learning. Third, the strength of unique cues should be strongest when training involves positive patterning, $\mathrm{AB}+, \mathrm{A}-, \mathrm{B}-$, as opposed to feature-positive, $\mathrm{AB}+, \mathrm{B}-$, training.

The results indicated that modality, temporal parameters, and presentations of the A feature alone interacted in various interesting ways. Most importantly, when training involved a serial procedure, the use of samemodality stimuli generally interfered with the rate of learning. If a configural strategy had been used to solve the discrimination, one might have expected better learning under this condition. In addition, although presentations of the feature alone did not affect the rate of acquisition, patterning improved transfer to the target from the alternate discrimination. This result is consistent with data from Holland (1989b) and with Holland's (e.g., $1983,1989 \mathrm{~b}$ ) suggestion that occasion setting is promoted by conditions that encourage a parsing of the stimuli on compound trials.

On the other hand, a simultaneous compound procedure produced a different pattern of results. First, the patterning discrimination was solved more slowly than the feature-positive discrimination. This result would be anticipated to the extent that simultaneous feature-positive discriminations are solved through simple excitatory feature-US associations. Since both stimuli are treated equivalently in the patterning procedure, a solution that involves simple excitation by either stimulus alone is precluded. Second, with $\mathrm{AB}+, \mathrm{A}-, \mathrm{B}-$ patterning training, the use of stimuli from the same modality for the feature and target enhanced the rate of learning. This was not the case with $\mathrm{AB}+, \mathrm{A}-$ feature-positive training. Given the assumption that configuring is encouraged with same-modality compounds, this result suggests that simultaneous patterning was solved through a configural mechanism.

Although complex, the results from these two experiments generally support Holland's early contention that the mechanism used in the solution of feature-positive and patterning discriminations depends largely on the procedures used during training. Most importantly, evidence of modulation was strongest under conditions that discouraged the use of configural cues. However, neither a simple associative nor a configural or unique cue perspective could account adequately for the overall pattern of results.

In general, given the abundant evidence of transfer within and across modality and response form, there is little support for the possibility that modulation is based entirely on a CS-specific mechanism. However, the incomplete transfer observed in most studies, as well as the finding that modality does affect the nature of the mechanism, should be taken as strong evidence favoring the contribution of some CS-specific process. Other evidence has been obtained in two recent investigations by Rescorla (1991a, 1991c), in which the extent of transfer across target CSs was used to assess the role of the orig- 
inal target CS. In one set of studies, Rescorla (1991c) examined the extent to which transfer is affected by posttraining manipulations of the original target CS-US associations. With positive, $\mathrm{AB}+, \mathrm{B}-$, and negative, $\mathrm{AB}-, \mathrm{B}+$, modulation procedures, Rescorla found that manipulations of the original B-US associations changed the extent to which A controlled responding in a transfer test. These results suggested that part of A's action on the transfer target was mediated by the associative character of $B$.

Another set of studies (Rescorla, 1991a; see also Morell \& Holland, 1993) assessed the importance of the original target identity by examining how modulators that had been trained with either the same or different targets would combine when tested on a common transfer target. If part of the modulatory effect on a transfer target is based on the original training target identity, the extent to which combinations of modulators control responding on a transfer test should depend on whether or not the modulators shared a common target during training. Indeed, modulators with a common training target combined to produce stronger transfer than did modulators that had been trained with different targets. The results of both of these sets of studies provide support for the contribution of a CS-specific modulatory process.

\section{Are the Positive and Negative Modulatory Roles Mutually Exclusive?}

Although certain observations imply a partial role for the original target in modulation, the extent to which modulation is independent of the CS may reflect the modulator's ability to act directly on some feature of the US. Rescorla (e.g., 1985, 1987) has frequently advocated this position and has further suggested that positive and negative modulators may have functionally opposite effects on the US. This view stems naturally from the procedural parallels involved in feature-negative, $\mathrm{AB}-, \mathrm{B}+$, and feature-positive, $\mathrm{AB}+, \mathrm{B}-$, training paradigms. In both procedures, reinforcement of $B$ depends on the presence of A. Appropriate responding to B may thus result from A's impact on the US representation evoked by $B$.

The view that positive and negative modulation are functionally opposing processes is encouraged by Rescorla's (1987) observation that positive and negative modulators have opposite effects on responding to a single target. Evidence for an opposing function also comes from an experiment that directly examined the impact of a positive modulator on a target that had a known inhibitory component. Rescorla (1987; but see Holland, 1986b) found that a positive modulator, but not an excitor, enhanced responding to the "AB" compound from an $\mathrm{AB}-, \mathrm{B}+$ Pavlovian conditioned inhibition discrimination. The modulator did not enhance responding to a compound of two "neutral" stimuli. Thus, the positive modulator appeared to reduce the control exerted by the inhibitor.

Another technique that has been used to assess the oppositional nature of positive and negative modulatory learning has been to examine variables that might affect acquisition, extinction, or expression of the modulation.
For example, if positive and negative modulators are to be characterized as stimuli that change the threshold for activation of the US representation in opposite directions, one might expect the two processes to show mutual interference in both learning and performance. Several experiments have explored the notion that positive and negative modulators possess opposite functional roles by examining the effect that training one type of modulation has on the acquisition or expression of the other modulatory property.

\section{Pretraining and Posttraining Manipulations}

Rescorla (1986a) used the pigeon autoshaping preparation to examine the effect of various post training procedures on positive modulation. In several experiments, he first trained two diffuse stimuli to modulate responding to each of two keylight targets. Then he repeatedly presented a nonreinforced compound of one of the modulators and either a simple excitor or an original target keylight. In comparison with the untreated diffuse stimulus, both of these manipulations disrupted the diffuse stimulus' modulatory ability. As mentioned previously, however, nonreinforcement of the diffuse stimulus in the absence of an excitatory target had no such decremental effect. To the extent that nonreinforcement of an excitatory keylight in the presence of a diffuse stimulus is a procedure that might otherwise produce negative modulatory control, these results further suggest that positive and negative modulation oppose one another.

Rescorla (1987) has also examined the effect of pretraining positive modulation on the acquisition of negative modulation. In one experiment, he trained one diffuse stimulus as a positive modulator and another as an excitor and then trained them in compound as an inhibitor. He found that only the excitor acquired inhibitory control as a result of such training, suggesting that positive modulatory training retarded the acquisition of inhibition. Two additional experiments examined the effects of positive modulation or excitatory post training on established inhibitory control. He found that positive modulation disrupted inhibitory control to a greater degree than did excitatory training. These results are a persuasive demonstration that positive modulation may be closer to being an opposite of inhibition than is excitation. However, Rescorla's (1991b) recent observation that excitatory training can actually enhance the development of inhibition suggests that the differential effects that excitation and positive modulation have on inhibition may result from the augmenting effects of excitation rather than from the interfering effects of positive modulation.

In any case, the results of these studies are consistent with the possibility that positive and negative modulators are opposites. They have opposing functions in the control of responding to target stimuli; the disruption of positive modulatory strength is accomplished best by procedures that are thought to encourage negative modulation; and when compared with excitatory training, modulatory training both slows the acquisition of and disrupts already established negative modulation. 


\section{Manipulations During Training}

Although positive and negative modulation thus appear to have functionally opposite roles, note that the mutual interference seen in the function of positive and negative modulators does not require that the positive and negative modulatory functions are mutually exclusive. Several researchers have provided data suggesting that one stimulus might concurrently possess both positive and negative modulatory properties. Such evidence has been obtained by using a procedure in which a stimulus is trained concurrently as both a positive and a negative modulator for different target stimuli. It should be clear that a US threshold-shifting interpretation of positive and negative modulation would be hard pressed to explain the solution of such a discrimination solely in terms of modulation.

To investigate acquisition of this "ambiguous cue" role for modulators, Holland and his collaborators (Holland, 1991b; Holland \& Reeve, 1991; see also Wilson \& Pearce, 1989) have used a preparation involving a discrete trial barpressing procedure with rats, in which, on positive trials, leverpressing during a $5-\mathrm{sec}$ target stimulus was reinforced with sucrose; on negative trials, leverpresses during presentation of the target were nonrewarded. A modulatory stimulus, $A$, acted as a positive feature for one stimulus, $\mathrm{A} \rightarrow \mathrm{B}+, \mathrm{B}-$, and concurrently as a negative feature for another, $\mathrm{A} \rightarrow \mathrm{C}-, \mathrm{C}+$. As with Holland's appetitive observational procedure, compound trials involved a 5 -sec presentation of $\mathrm{A}$, followed after a 5-sec empty interval by the 5-sec target, B or C. (See Holland, 1991a, for a demonstration of both positive and negative occasion setting with this procedure.)

In one set of experiments, Holland and Reeve (1991) compared the rate of acquisition of ambiguous discrimination learning with that found in positive or negative patterning discriminations. The results showed that acquisition of modulation by the ambiguous modulator on both positive and negative trials was as fast as that acquired by the analogous modulator that was trained as either a positive or a negative modulator alone. This suggests that the concurrent positive and negative training do not show mutual interference.

To investigate the nature of the modulation acquired by the ambiguous cue, transfer to a target stimulus was examined while the stimulus was undergoing excitatory training and extinction. Although Holland and his collaborators had not previously observed transfer to an extinguished stimulus with the observational preparation with rats (e.g., Holland, 1986b), transfer of positive modulation has been observed with this discrete-trial preparation (Holland, 1991a). Indeed, with the present ambiguous training, the modulator enhanced responding to a CS that was currently undergoing extinction. However, the modulator did not affect responding during excitatory acquisition. This observation might suggest that positive modulation generalizes more broadly than does negative modulation. In any case, although the separate contributions of positive and negative modulatory control to transfer might oppose one another and would thus be dif- ficult to assess, the observation of transfer suggests that control by the ambiguous modulator is to some extent independent of the original target CSs.

The authors noted that there are several alternative processes through which the ambiguous discrimination could be solved. For example, both tasks could be solved through configuring. Alternatively, the ambiguous feature could signal nonreinforcement of one target through a negative modulatory mechanism, but form a unique configuration with the other target on reinforced compound trials. Conversely, positive modulation of responding during one target could coexist with the formation of a nonreinforced configuration with the other.

Another set of experiments (Holland, 1991b) addressed these possibilities by examining transfer of both positive and negative modulation to various targets following ambiguous training. All rats received ambiguous cue training; then, separate groups each received further training on various other discriminations, including training of a second ambiguous cue, feature-positive training, feature-negative training, or a "pseudo-discrimination" in which a cue was uninformative about reinforcement of the response. Results of the transfer tests revealed that the ambiguous cue modulated responding to the various targets in a manner consistent with the targets' training histories. The cue enhanced responding to the two targets that had been nonreinforced when presented alone, and it suppressed responding to the two targets that had been reinforced when presented alone. Likewise, the targets of the ambiguous cue were also modulated appropriately by the feature-positive and feature-negative modulators. In general, the observation of transfer suggested that solution of the discriminations was not entirely configural. Furthermore, appropriate responding to the targets appeared to depend on a specific property of the cues that was acquired during training.

Although the results of these transfer tests suggested that the ambiguous cue had both positive and negative modulatory properties, it is also possible that modulation of the targets was not entirely due to the training received by the cue. Wilson and Pearce (1989), for example, trained separate feature-positive and feature-negative discriminations, using an appetitive procedure with rats, and found that a novel cue modulated responding to the targets in the same manner as did the original features. Such generalization between the cues could account for Holland's transfer data as well. In a subsequent experiment, however, Holland (1991b) did not find ambiguous modulatory control by a simple excitatory stimulus, thus making it less likely that transfer had occurred entirely through generalization. Taken together, the results of these experiments suggest that not only are positive and negative modulation independent of simple associations with the US, under some circumstances, they may also be independent of one another.

The ambiguous cue experiments highlight an interesting issue that has received relatively little systematic investigation. The recent view espoused by Holland and his collaborators is that targets that have participated in 
positive or negative modulatory discriminations will be sensitive to control by positive or negative modulators other than that with which they were trained. However, an important feature of this view is that control is specific to the type of training experienced by the targets. For example, responding to targets of positive modulators will be enhanced by other positive modulators, but will not be suppressed by negative modulators. Although this assumption is perhaps difficult to test, it may provide some insight into the important properties of targets that allow them to come under modulatory control. Holland's recent observation that positive modulators (Holland, 1991a) and ambiguous cue modulators (Holland \& Reeve, 1991) enhance responding to extinguished excitors encourages the view advocated by other researchers that sensitivity to control may be influenced by factors other than prior modulatory training. For example, Rescorla (e.g., 1988) has suggested that extinguished stimuli and targets of positive modulators may both possess an inhibitory component that is sensitive to positive modulatory control.

Although the ambiguous cue results appear to contradict the suggestion that positive and negative modulation functionally oppose one another, it is important to bear in mind that in some situations control by positive and negative modulators is less specific to the targets on which they act. Mutual interference would be more likely under conditions that encourage transfer. Furthermore, it is conceivable that concurrent training establishes stronger CS specificity of both positive and negative control. It would be valuable to examine the generality of these findings across preparations such as autoshaping, where the specificity of control to the original targets is not as strong.

\section{Summary of the Different Modulatory Views}

It is important to emphasize that the general pattern of results supports the contribution of several different mechanisms to modulatory control. The possibility that modulators act on the US, perhaps by changing the threshold for activation of the US representation (e.g., Rescorla, 1985), is supported in large part by the results of transfer experiments. The further observation that the extent of modulatory transfer depends on the target's training history suggests that modulators come to act on some attribute of the US representation to which only certain target stimuli are sensitive. It is also likely, however, that generalization across targets or modulators contributes importantly to many instances of transfer. The model proposed by Pearce (1987) provides considerable insight into the potential role of generalization in modulation. The likelihood that the identity of the original training CS is encoded during modulation training is further supported by incomplete transfer across targets, and by the results of Rescorla's (1991a, 1991c) recent experiments demonstrating that transfer is mediated in part by the identity and current associative value of the training target CS. Moreover, a further elaboration of Pearce's (1987) model that provides for generalization based on the training history of the stimuli could explain a substantial number of transfer findings that cannot be readily explained through generalization between the perceptual features of the stimuli alone. Although speculative, such an account would support Holland's (1983) early CS-specific view of modulation, while allowing transfer under some circumstances. What remains to be discovered, however, are the types of conditioning treatments that would enhance generalization between perceptually dissimilar stimuli.

Finally, the recent research from Holland's laboratory investigating the nature of ambiguous cue learning suggests that modulatory functions can arise under conditions that discourage the use of a more general US-based mechanism or strategy. This research is likely to provide important information about the associative structure of the targets involved in modulation and transfer.

\section{WHEN DOES MODULATION OCCUR?}

One of the most important issues to be addressed concerns the conditions that are necessary for a stimulus to acquire a modulatory function.

\section{The Importance of the Modulator-Target Interval}

\section{Positive Modulation}

Much of the early research on both positive and negative modulation from Holland's laboratory emphasized the importance of serial $\mathrm{A} \rightarrow \mathrm{B}$, as opposed to simultaneous, $\mathrm{AB}$, compound presentations in establishing a modulatory capacity for $\mathrm{A}$. For example, examining positive modulation with the behavioral observation technique, Holland (1986a, see also Ross \& Holland, 1981) systematically varied the durations of the light $A$ and tone $B$, and the relationship of the light's termination to the onset of B. Positive modulation, defined as greater headjerk responding to the tone in compound with the light than to the tone alone, was generally strongest with longer light durations. Furthermore, modulation was strengthened by the insertion of an empty trace interval between the termination of the light and onset of the tone. Because excitation to the light feature would be expected to decline as the interval between the light's termination and the US increases, these results provided support for the independence of modulation and excitation and set the stage for an examination of a modulatory mechanism independent from excitation.

Although several sets of findings discussed above have supported an important role for temporal parameters in positive modulation, Holland $(1989 \mathrm{c})$ has recently examined the effects of manipulating the relative salience of the feature and target $\mathrm{CS}$ on solution of a simultaneous, $\mathrm{AB}+, \mathrm{B}-$, feature-positive discrimination. When B was sufficiently salient so as to acquire excitation, this excitatory responding was modulated by $\mathrm{A}$. Modulation was thus demonstrated with a simultaneous procedure. Subsequent presentations of A alone did not disrupt modulation, suggesting that the modulation did not depend on A's excitatory strength. Furthermore, con- 
trary to the findings of Rescorla (1986b), conditioning excitation to $B$ prior to discrimination training did not promote a modulatory role for $\mathrm{A}$. Thus, although the early work indicated the importance of $A \rightarrow B$ temporal asynchrony, these results encourage the possibility that serial arrangements may have an impact by providing a differential level of salience to the feature and target.

Looney and Griffin (1978) have also obtained results suggesting that the $\mathrm{AB}$ temporal relationship can affect the learning of a feature-positive discrimination. Using pigeons in an autoshaping preparation, they found that a simultaneous $\mathrm{AB}+, \mathrm{B}-$ discrimination was solved more slowly than a serial $A \rightarrow B+, B-$ discrimination when $A$ was a tone and $B$ was a keylight. Although they did not investigate a possible modulatory role for the tone, the results are consistent with the possibility that the tone blocked learning about the keylight during simultaneous compound training. Serial training may have attenuated excitatory conditioning of the tone feature.

Thus, some evidence suggests that serial procedures may encourage modulation by allowing the target to acquire the necessary excitatory association with the US. However, it also seems likely that serial training, and perhaps differential salience of the modulator and target, may also encourage a "perceptual discontinuity" between the feature and target (e.g., Holland, 1986a), reducing the potential for perceiving the stimuli as a single configured stimulus.

\section{Negative Modulation}

The importance of the temporal procedures to the nature of inhibitory learning is an issue that has received considerable attention. Holland and his collaborators (e.g., Holland, 1984; Holland \& Gory, 1986; Holland \& Lamarre, 1984) have found several instances in which serially and simultaneously trained inhibitors have different properties. They suggest that with simultaneous $\mathrm{AB}-, \mathrm{B}+$ procedures, A's inhibitory control may be based on direct inhibitory associations with the US, opposite to those acquired during excitatory training. With serial compounds, A controls hierarchically by suppressing the activation of the B-US association. Rescorla (e.g., $1979,1985,1989$ ), on the other hand, has espoused the view that, regardless of the temporal procedures, inhibitors may act on the US by raising its activation threshold.

The distinction between simple associative inhibitory mechanisms and modulatory inhibitory mechanisms appears to rely heavily on the traditional assumption that an inhibitory CS cannot otherwise be excitatory. All stimuli must lie somewhere on the continuum of associative strength. The finding that inhibitory control can coexist with excitation encouraged the view that some types of inhibitory control must involve a fundamentally different mechanism. In fact, recent data from several different preparations suggest that it is common for excitatory associations to mask the full extent of inhibitory control. In situations where the inhibitor's excitatory CR interacts with that evoked by the target used for inhibitory assessment, the magnitude of underlying inhibition can be un- derestimated (Cunningham, 1981; DeVito \& Fowler, 1987; Holland, 1984; Rescorla, 1982b, Williams \& Overmier, 1988). Under some conditions, the expression of coexisting excitation or inhibition may depend on the method used to assess these properties (e.g., Matzel, Gladstein, \& Miller, 1988; Tait \& Saladin, 1986). Williams, Overmier, and LoLordo (1992) present an excellent summary and elaboration of these issues. In general, results like these suggest that the traditional conception of inhibition and excitation as mutually exclusive properties is probably an oversimplification. If inhibition and excitation can coexist, it becomes worthwhile to question altogether whether they should be characterized as involving the same mechanism.

Holland's suggestion that simultaneous and serial feature-negative procedures produce different types of inhibitory control is based on the results of several manipulations. First, Holland (1984) found that simultaneous, but not serially trained, inhibitory control is disrupted by excitation when testing is conducted with the temporal parameters used in training. Although these data are the most substantial, bear in mind the importance of testing differently trained stimuli with identical testing procedures. The most convincing data must come from studies in which both types of inhibitors are tested either serially or simultaneously. Although the inhibitor's excitatory $C R$ would be expected to interfere with the expression of inhibition during simultaneous tests, Holland (1984), using conditioned suppression with rats, and Holland (199 la), using the operant discrete-trial barpressing preparation with rats, provide data with simultaneous tests in which a serially trained inhibitor retained some of its inhibitory control following excitatory conditioning, whereas a simultaneously trained inhibitor's control was disrupted. These data support the contention that simultaneously trained inhibition is not compatible with excitation.

With serial tests, excitatory CRs should occur prior to presentation of the target and might therefore be less likely to interfere with inhibitory control. Serially tested control by serially trained inhibitors is not disrupted by the presence of excitatory CRs (Holland, 1984). Unfortunately, as mentioned previously, inhibition by simultaneously trained inhibitors is often considerably reduced if not eliminated with serial testing (Detke, 1991; Holland, 1984, 1989d; Holland \& Lamarre, 1984; Rescorla, 1989; but see Holland \& Gory, 1986). Although the reason for this loss is unclear, it reduces the effectiveness of serial tests for examining the influence of excitation on simultaneously trained inhibitors.

A second method for examining the effect of excitation on underlying inhibition is to reassess inhibitory control after extinguishing any excitatory CRs. A traditional associative account of inhibition would predict that excitatory training of an inhibitor should abolish the inhibition; extinction of this excitation should leave the stimulus associatively neutral. Holland (1984; see also Rescorla, 1985) used this technique to examine the effect of excitatory conditioning on a simultaneously trained 
inhibitor. Importantly, he observed that even though simultaneously trained inhibition was disrupted by excitatory training, subsequent extinction of the excitatory $\mathrm{CR}$ exposed the presence of underlying inhibition. Thus, with circumstances under which inhibitory control is removed by excitation, the underlying inhibition can remain intact. These data are somewhat troubling for Holland's contention that simultaneously trained inhibition is lost following excitatory training.

A third line of evidence suggesting multiple inhibitory processes is that simultaneously but not serially trained inhibitors show retarded rates of excitatory conditioning (e.g., Holland, 1984; Holland, 1989d). However, one should bear in mind that serial inhibitors often possess considerable second-order excitation during inhibitory training (e.g., Holland, 1984; Holland \& Lamarre, 1984; Rescorla, 1982b). Thus, the faster rate of conditioning in these groups may reflect a savings of excitatory response strength.

The fourth major dissociation comes from the effects of nonreinforced exposure on inhibition. Holland and Gory (1986) found that a serially trained inhibitor will show weaker inhibitory control if presented alone following inhibitory training. Extinction of simultaneously trained inhibitors, on the other hand, has sometimes been found to result in enhanced inhibitory control (e.g., DeVito \& Fowler, 1987; Holland \& Gory, 1986; Williams \& Overmier, 1988; Williams, Travis, \& Overmier, 1986). Although the existing accounts of modulation do not predict extinction of serially trained inhibition per se, the observation further suggests that different procedures produce different types of inhibitors.

Although fundamentally different mechanisms might underlie the difference between simultaneous and serial inhibitors, the evidence is also consistent with the possibility that the two training procedures produce inhibitors that use a similar mechanism but that have different associative structures. Although simultaneous training and testing typically results in stronger inhibitory control, serially trained stimuli acquire stronger within-compound and second-order associations. Holland and Gory (1986) suggested the possibility that these excitatory CRs may play an inhibitory cuing role. If excitation plays an inhibitory role with serially trained inhibitors, further excitatory conditioning would not be expected to disrupt inhibition, excitatory conditioning of the inhibitor should be rapid, and extinction of the inhibitor's excitation should disrupt inhibition. Thus, such a possibility could go a long way toward explaining some of the differences between serially and simultaneously trained inhibitors. However, note that extinction of simultaneously trained inhibitors can sometimes enhance inhibition. Thus, a complete picture might require one to assume that excitation acquired during simultaneous training interferes with the expression of inhibition, whereas excitation acquired serially is an important part of the inhibitory complex.

Whereas many experiments have been focused on the differences in the underlying inhibitory mechanisms,
Rescorla (1989) has suggested that it is also important to consider the degree to which the different training procedures establish different magnitudes of inhibitory control. The strength of inhibitory control may be largely determined by the treatment received by the excitatory target used for inhibitory training. In simultaneous training, the target is reinforced whenever it is presented alone, and it is nonreinforced only during simultaneous compounds with the feature. In serial training, however, the target is presented alone on both reinforced and nonreinforced trials. This distinction may make the target differentially effective in establishing inhibition. In order to compare the inhibitory control acquired through serial and simultaneous procedures, Rescorla argued that the target of the two inhibitors should be otherwise treated identically. In two autoshaping experiments, he compared the strength of serially and simultaneously trained inhibition when both training and testing involved a common excitatory target, and when testing involved common temporal procedures. The results revealed that although inhibitory control was weaker with serial compounds during both training and testing, the magnitude of inhibition under common testing conditions did not depend on the temporal procedures involved in training.

Because the target's treatment was held constant, this experiment suggests that at least part of the distinction observed between simultaneous and serial training may be due to the effectiveness of the target in establishing inhibition. On the other hand, the effect of training different types of inhibitors with a common target has received very little systematic investigation. It is therefore important to recognize that Rescorla's (1989) use of a common target for both simultaneous and serial training may have affected the nature of the inhibitory learning that occurred, perhaps through increased generalization across the inhibitors (see also Detke, 1991).

In general, there are a number of situations in which serial and simultaneously trained inhibitors show different properties and are differentially affected by various manipulations. However, a difference in the inhibitory function of a stimulus following different training procedures does not necessarily imply that there is a difference in the basic learning mechanisms. Rather, the functional differences may result from differences in the role that excitation plays and in the magnitudes of inhibitory control. Inhibitory control may involve a process that is relatively independent of excitation regardless of the inhibitory training procedures.

\section{Informational Value}

Most researchers share the view that a necessary condition for modulation is that the modulator provide information about target reinforcement. This could be inferred from the fact that almost all of the recent research on Pavlovian modulation has employed discrimination paradigms-feature-positive, feature-negative, patterning, or conditional discrimination. With each procedure, the stimuli are at least theoretically capable of providing in- 
formation about reinforcement of the other stimulus. This viewpoint is perhaps best exemplified by the use of the pseudofacilitator, or $\mathrm{AB}+, \mathrm{B}+$, treatment as a control for positive modulation acquired through $\mathrm{AB}+, \mathrm{B}-$ discrimination training (see, e.g., Davidson et al., 1988; Davidson \& Rescorla, 1986; Holland, 1986b; Rescorla, 1985; Wilson \& Pearce, 1989). Although the pseudofacilitator may not control accurately for the modulator's excitation or for the associative status of the modulator's target, the pseudofacilitator and modulator are paired an equal number of times with a target and with the US. Only the modulator provides information about target reinforcement. Provided that the role of excitation is convincingly ruled out through other experiments or procedures, the consistent observation that the pseudofacilitator does not acquire a modulatory ability suggests that modulation depends on the information provided by the feature.

Several researchers have addressed the importance of the informational value of modulators by examining whether modulators follow the same rules of competition that are thought to exist between Pavlovian excitatory stimuli. For example, the popular conception of simple associative learning is that excitation is acquired to the extent that a stimulus provides information about the occurrence of the US (e.g., Rescorla \& Wagner, 1972). Bonardi (1991; see also LoLordo \& Ross, 1987, and Ross \& LoLordo, 1986; but see LoLordo \& Ross, 1990) has recently provided some evidence that a pretrained positive modulator will "block" the acquisition of the ability to modulate by another stimulus that provides no new information about target reinforcement. The experiments involved a modulation training procedure in which a 5-sec auditory stimulus (B) was reinforced with food when presented during a 3-min visual or auditory feature stimulus (A). When $B$ was presented alone, it was nonreinforced. A control group received $B$ and food quasirandomly during $\mathrm{A}$. In the blocking phase, both groups received the modulatory training, but the modulator was now a simultaneous compound of the original A stimulus and an added auditory or visual $\mathrm{C}$ stimulus. A test session then assessed the ability of $\mathrm{C}$ alone to enhance responding to B. Results showed that pretraining with A produced nearly complete blocking of the acquisition of modulation by $\mathrm{C}$ in the experimental group, whereas the control group showed strong modulation by $\mathrm{C}$. Bonardi found similar results in a second experiment that used a within-subjects design.

The observation of blocking further suggests that modulatory acquisition depends on the degree to which the to-be-trained modulator provides information about the reinforcement of the target. If target reinforcement is already signaled by one modulator, the added signal will be informationally redundant and thus will not acquire the modulatory ability.

\section{Positive Modulation Based on Inhibition}

In the casual account of modulatory acquisition, the modulator plays an active role in solution of a discrimi- nation by providing information about target reinforcement. Rescorla (1988) has suggested an alternative account in which the feature acquires modulatory properties in a more passive manner. The view is based in part on the evidence that positive and negative modulation may be opposite processes. The observations that positive modulation training disrupts inhibitory control (Rescorla, 1987) and vice versa (Rescorla, 1986a), and the finding that positive modulators act best on targets that may be thought to have some inhibitory component, such as extinguished stimuli, might suggest that positive modulators act by reversing the effects of inhibition.

If positive modulation opposes inhibition, Rescorla (1988) reasoned, modulation might be acquired best when a target with an inhibitory component undergoes reinforcement. In two experiments, he pretrained keylights that would later be used as targets. One was trained as a conditioned inhibitor of another keylight; the other was presented alone. Two diffuse stimuli were then trained as positive modulators for one of the two pretrained targets. When modulation was tested on the excitor from the original inhibition pretraining, the modulator whose target was originally inhibitory was more effective than the stimulus whose target had been preexposed. In a third experiment, Rescorla used targets that had been pretrained as either the inhibitor or the excitor from a Pavlovian conditioned inhibition procedure. Again, positive modulation was stronger with the modulator whose target was inhibitory. Rescorla suggested that these results are consistent with the view that positive modulation is acquired when an inhibitory stimulus is reinforced.

The possibility that positive modulation depends on the reinforcement of a stimulus with an inhibitory component further suggests that positive modulation represents an acquired ability to suppress an ongoing inhibitory process. This view is consistent with the observation that positive modulatory control depends critically on the training history of the target. By suppressing inhibition, positive modulators may expose the target's underlying excitation. Although these ideas are still somewhat speculative, this experiment is an important step in our understanding of the conditions under which modulation is acquired.

\section{CONCLUSION}

The recent interest in Pavlovian modulation has been stimulated in large part by evidence that is not easily explained through a system that consists solely of simple associations. Pavlovian learning appears to involve a mechanism whereby the evocation of CRs can depend, not on the CS-US association alone, but on additional stimuli that modulate the expression of the association.

In an effort to better understand the role that modulation plays in learning, investigators have examined the properties of modulators, the interaction of positive and negative modulators, and the relationship between the modulatory function and the simple associative func- 
tion. Little has yet been said, however, about the possible functions of modulatory control in learning. One speculation is that the modulatory mechanism allows the animal to retain a representation of CS reinforcement despite episodes of extinction. Situations such as this, where stimulus-reinforcer contingencies are not consistent, might encourage the animal to make use of other stimuli to resolve the ambiguity (see Bouton, 1991). On the one hand, popular associative theories suggest that associative strength is acquired by the best predictor of reinforcement. For example, any excitation that is initially acquired by the target stimulus $B$ of an $\mathrm{AB}+, \mathrm{B}$ - discrimination would decrease as $\mathrm{A}$ became more excitatory. However, in a modulatory account emphasizing the retention of a CS's training history, B's excitation would remain intact. As a result of training, responding to $\mathrm{B}$ becomes dependent on the presence of $\mathrm{A}$. The animal thus retains the reinforced representation of $B$ and uses information provided by $\mathrm{A}$ as a signal for its activation.

Such an account obviously involves a more complex structure than is required by traditional associative models. However, a modulatory mechanism allows the animal to retain a more complete account of its training experience than is afforded by simple associations alone.

The observation that modulators control the expression of CS-US associations relatively independently from their own US associations is consistent with similar findings from investigations of the role of contextual or background stimuli in learning. The results of several series of experiments using both aversive and appetitive preparations with rats have revealed a number of similarities between Pavlovian modulators and contexts (e.g., Bouton \& Swartzentruber, 1986; Grahame, Hallam, Geier, \& Miller, 1990; Swartzentruber, 1991, 1993; Swartzentruber \& Bouton, 1988). Bouton and Swartzentruber (1986), for example, used a conditioned suppression preparation with rats to examine parallels between contextual control of responding to CSs and modulatory control by punctate stimuli. Our contexts differed from each other in their visual features, the odors present, the grid floors, and shape of the lever used for operant responding. In several experiments, we trained contextual discriminations in which a tone CS was paired with shock during sessions in Context $A$ and nonreinforced during sessions in Context B. Within several two-session cycles, freezing was much stronger during the tone in $\mathrm{A}$ than in $\mathrm{B}$. We then conducted tests to assess the associative properties of the contexts. Compared with control groups that received equal exposure to the contexts but that lacked context discrimination training, neither Context A nor Context B showed any reliable evidence of excitatory or inhibitory associations with the US, and nonreinforced exposure to Context A did not reduce the extent of contextual control. Thus, as with modulation by punctate cues, the contexts controlled responding to the CSs in a manner that appeared to be independent of direct US associations.
More recently, I have used Holland's observational procedures with rats to examine blocking of modulatory control between contexts and traditional modulators (Swartzentruber, 1991). In the first phase of one experiment, I trained a serial feature-positive discrimination, $\mathrm{L} \rightarrow \mathrm{T}+, \mathrm{T}-$, in which a light, $\mathrm{L}$, was trained as a signal for reinforcement of a tone, $T$. Presentations of $T$ alone were nonreinforced. In the second phase, the $\mathrm{L} \rightarrow \mathrm{T}+$ compound trials were presented in Context $\mathrm{A}$, and $\mathrm{T}-$ trials were presented in Context $\mathrm{B}$. Thus, further training was conducted in a manner that would allow the contexts to acquire control of responding to the tone. When comparisons were made with groups that received no prior modulatory training with the light, the light blocked learning about Context $\mathrm{A}$ in the second phase. A second experiment examined the converse situation; a context that signaled reinforcement of the tone blocked acquisition of modulatory control by the light.

The results of these experiments provide a fairly convincing argument that animals can use contextual stimuli to modulate the expression of CS-US associations. As with modulation by punctate stimuli described earlier, contexts modulate in a manner that cannot be easily explained through the existence of direct associations with the US alone. Moreover, the evidence for reciprocal blocking suggests that contexts and punctate modulators use the same mechanism in control of responding to target CSs. Modulatory control by contextual stimuli may be involved in a number of situations in which CSs are treated differently in different contexts. Bouton (e.g., 1991) has suggested that contexts and punctate modulators may both work by retrieving separate memories of CS reinforcement and nonreinforcement. In any case, it is easy to see the adaptive value of a mechanism that selectively activates individual associations following training with multiple incompatible episodes.

In general, an informational occasion-setting role like the one that Skinner (1938) advocated for an instrumental discriminative stimulus appears to be at work in Pavlovian preparations. In situations in which a CS has been both reinforced and nonreinforced, modulators influence the extent of conditioned responding. Moreover, the resemblance of Pavlovian modulation to instrumental discriminative stimulus control may go beyond the procedural similarities. Davidson et al. (1988) found that a stimulus trained to signal reinforcement of an instrumental response also enhanced responding to a target of a Pavlovian positive modulator. Similarly, the positive modulator reliably increased the probability of the instrumental response. Results such as these suggest that Pavlovian and instrumental modulators may function through a shared mechanism.

Finally, although the existence of some sort of Pavlovian modulatory mechanism seems to be established, we still know little about the necessary conditions under which modulation is acquired. Forthcoming experiments addressing this issue will perhaps provide the greatest insight into the role that modulation plays in learning. 


\section{REFERENCES}

ARnold, H. M., Grahame, N. J., \& Miller, R. R. (1991). Higher order occasion setting. Animal Learning \& Behavior, 19, 58-64.

BombaCE, J. C., BRANDON, S. E., \& Wagner, A. R. (1991). Modulation of a conditioned eyeblink response by a putative emotive stimulus conditioned with hindleg shock. Journal of Experimental Psychology: Animal Behavior Processes, 17, 323-333.

BONARDI, C. (1991). Blocking of occasion setting in feature-positive discriminations. Quarterly Journal of Experimental Psychology, 43B, $431-448$

Bouton, M. E. (1991). Context and retrieval in extinction and in other examples of interference in simple associative learning. In L. W. Dachowski \& C. F. Flaherty (Eds.), Current topics in animal learning: Brain, emotion, and cognition (pp. 25-53). Hillsdale, NJ: Erlbaum.

Bouton, M. E., \& Swartzentruber, D. (1986). Analysis of the associative and occasion-setting properties of contexts participating in a Pavlovian discrimination. Journal of Experimental Psychology: Animal Behavior Processes, 12, 333-350.

Brandon, S. E., Bombace, J. C., Falls, W. A., \& Wagner, A. R. (1991). Modulation of unconditioned defensive reflexes by a putative emotive Pavlovian conditioned stimulus. Journal of Experimental Psychology: Animal Behavior Processes, 17, 312-322.

Brandon, S. E., \& WAGNER, A. R. (1991). Modulation of a discrete Pavlovian conditioned reflex by a putative emotive Pavlovian conditioned stimulus. Journal of Experimental Psychology: Animal Behavior Processes, 17, 299-311.

CunNingham, C. L. (1981). Association between the elements of a bivalent compound stimulus. Journal of Experimental Psychology: Animal Behavior Processes, 7, 425-436.

Davidson, T. L., Aparicio, J., \& Rescorla, R. A. (1988). Transfer between Pavlovian facilitators and instrumental discriminative stimuli. Animal Learning \& Behavior, 16, 285-291.

Davidson, T. L., \& JARRARD, L. E. (1989). Retention of concurrent conditional discriminations in rats with ibotenate lesions of hippocampus. Psychobiology, 17, 49-60.

Davidson, T. L., \& Rescorla, R. A. (1986). Transfer of facilitation in the rat. Animal Learning \& Behavior, 14, 380-386.

DETKE, M. J. (1991). Extinction of sequential conditioned inhibition. Animal Learning \& Behavior, 19, 345-354.

DEVITO, P. L., \& FowLER, H. (1987). Enhancement of conditioned inhibition via an extinction treatment. Animal Learning \& Behavior, $15,448-454$

Grahame, N. J., Hallam, S. C., Geier, L., \& Miller, R. R. (1990) Context as an occasion setter following either CS acquisition and extinction or CS acquisition alone. Learning \& Motivation, 21, 237. 265 .

HeARST, E. (1978). Stimulus relationships and feature selection in learning and behavior. In S. H. Hulse, H. Fowler, \& W. K. Honig (Eds.), Cognitive processes in animal behavior (pp. 51-88). Hillsdale, NJ: Erlbaum.

HEARST, E. (1984). Absence as information: Some implications for learning, performance, and representational processes. In $\mathrm{H}$. L. Roitblat, T. G. Bever, \& H. S. Terrace (Eds.), Animal cognition (pp. 311-332). Hillsdale, NJ: Erlbaum.

Holland, P. C. (1977). Conditioned stimulus as a determinant of the form of the Pavlovian conditioned response. Journal of Experimental Psychology: Animal Behavior Processes, 3, 77-104.

Holland, P. C. (1983). Occasion setting in Pavlovian feature positive discriminations. In M. L. Commons, R. J. Herrnstein, \& A. R. Wagner (Eds.), Quantitative analyses of behavior: Discrimination processes (Vol. 4, pp. 183-206). New York: Ballinger.

Holland, P. C. (1984). Differential effects of reinforcement of an inhibitory feature after serial and simultaneous feature negative discrimination training. Journal of Experimental Psychology: Animal Behavior Processes, 10, 461-475.

Holland, P. C. (1985). The nature of conditioned inhibition in serial and simultaneous feature negative discriminations. In R. R. Miller \& N. E. Spear (Eds.), Information processing in animals: Conditioned inhibition (pp. 267-297). Hillsdale, NJ: Erlbaum.

HollaND, P. C. (1986a). Temporal determinants of occasion setting in feature-positive discriminations. Animal Learning \& Behavior, 14 111-120.

Holland, P. C. (1986b). Transfer after serial feature positive discrimination training. Learning \& Motivation, 17, 243-268.

Holland, P. C. (1989a). Acquisition and transfer of conditional discrimination performance. Journal of Experimental Psychology: Animal Behavior Processes, 15, 154-165.

Holland, P. C. (1989b). Feature extinction enhances transfer of occasion setting. Animal Learning \& Behavior, 17, 269-279.

HollaNd, P. C. (1989c). Occasion setting with simultaneous compounds in rats. Journal of Experimental Psychology: Animal Behavior Processes, 15, 183-193.

Holland, P. C. (1989d). Transfer of negative occasion setting and conditioned inhibition across conditioned and unconditioned stimuli. Journal of Experimental Psychology: Animal Behavior Processes, 15, 311-328.

Holland, P. C. (1991a), Acquisition and transfer of occasion setting in operant feature positive and feature negative discriminations. Learning \& Motivation, 22, 366-387.

Holland, P. C. (1991b). Transfer of control in ambiguous discriminations. Journal of Experimental Psychology: Animal Behavior Processes, 17, 231-248.

HollaNd, P. C., \& BLOCK, H. (1983). Evidence for a unique cue in positive patterning. Bulletin of the Psychonomic Society, 21, 297-300.

Holland, P. C., \& Coldwell, S. E. (1993). Transfer of inhibitory stimulus control in operant feature-negative discriminations. Learning \& Motivation, 24, 345-375.

Holland, P. C., \& GoRY, J. (1986). Extinction of inhibition after serial and simultaneous feature negative discrimination training. Quarterly Journal of Experimental Psychology, 38B, 245-265.

Holland, P. C., \& LAMARRE, J. (1984). Transfer of inhibition after serial and simultaneous feature negative discrimination training. Learning \& Motivation, 15, 219-243.

Holland, P. C., \& Reeve, C. E. (1991). Acquisition and transfer of control by an ambiguous cue. Animal Learning \& Behavior, 19 , 113-124.

Honey, R. C., \& HALL, G. (1989). Acquired equivalence and distinctiveness of cues. Journal of Experimental Psychology: Animal Behavior Processes, 15, 338-346.

JARRARD, L. E., \& DAvidSON, T. L. (1991). On the hippocampus and learned conditional responding: Effects of aspiration versus ibotenate lesions. Hippocampus, 1, 107-117.

JENKINS, H. M. (1985). Conditioned inhibition of key pecking in the pigeon. In R. R. Miller \& N. E. Spear (Eds.), Information processing in animals: Conditioned inhibition (pp. 327-354). Hillsdale, NJ: Erlbaum.

KaPLAN, P. S., \& HeArst, E. (1982). Bridging temporal gaps between CS and US in autoshaping: Insertion of other stimuli before, during, and after CS. Journal of Experimental Psychology: Animal Behavior Processes, 8, 187-203.

Kehoe, E. J., Gibbs, C. M., Garcia, E., \& Gormezano, I. (1979). Associative transfer and stimulus selection in classical conditioning of the rabbit's nictitating membrane response to serial compound CSs. Journal of Experimental Psychology: Animal Behavior Processes, 5, 1-19.

KonORSKI, J. (1948). Conditioned reflexes and neuron organization. Cambridge: Cambridge University Press.

KONORSKI, J. (1967). Integrative activity of the brain. Chicago: University of Chicago Press.

LAMARRE, J., \& Holland, P. C. (1987). Transfer of inhibition after serial feature negative discrimination training. Learning \& Motivation, 18, 319-342.

LoLORDO, V. M., \& Ross, R. T. (1987). Role of within-compound associations in occasion setting: A blocking analysis. Journal of Experimental Psychology: Animal Behavior Processes, 13, 156-167.

LoLoRDo, V. M., \& Ross, R. T. (1990). Retraction of Ross and LoLordo findings concerning blocking in serial feature-positive discriminations. Journal of Experimental Psychology: Animal Behavior Processes, 16, 402-406.

LOONEY, T. A., \& GRIFFIN, R. W. (1978). A sequential feature-positive effect using tone as the distinguishing feature in an autoshaping procedure. Animal Learning \& Behavior, 6, 401-405. 
Matzel, L. D., Gladstein, L., \& Miller, R. R. (1988). Conditioned excitation and conditioned inhibition are not mutually exclusive. Learning \& Motivation, 19, 99-121.

Morell, J. R., \& Holland, P. C. (1993). Summation and transfer of negative occasion setting. Animal Learning \& Behavior, 21, 145-153.

Pearce, J. M. (1987). A model for stimulus generalization in Pavlovian conditioning. Psychological Review, 94, 61-73.

Pearce, J. M., Nicholas, D. J., \& Dickinson, A. (1981). The potentiation effect during serial conditioning. Quarterly Journal of Experimental Psychology, 33B, 159-179.

Pearce, J. M., \& Wilson, P. N. (1991). Failure of excitatory conditioning to extinguish the influence of a conditioned inhibitor. Journal of Experimental Psychology: Animal Behavior Processes, 17, 519-529.

REsCorla, R. A. (1969). Pavlovian conditioned inhibition. Psychological Bulletin, 72, 77-94.

RESCORLA, R. A. (1973). Evidence for "unique stimulus" account of configural conditioning. Journal of Comparative \& Physiological Psychology, 85, 331-338.

RESCORLA, R. A. (1979). Conditioned inhibition and extinction. In A. Dickinson \& R. A. Boakes (Eds.), Mechanisms of learning and motivation: A memorial volume to Jerzy Konorski (pp. 83-110). Hillsdale, NJ: Erlbaum.

RESCORLA, R. A. (1982a). Effect of a stimulus intervening between CS and US in autoshaping. Journal of Experimental Psychology: Animal Behavior Processes, 8, 131-141.

RESCORLA, R. A. (1982b). Some consequences of associations between the excitor and the inhibitor in a conditioned inhibition paradigm. Journal of Experimental Psychology: Animal Behavior Processes, 8 , 288-298.

Rescorla, R. A. (1985). Conditioned inhibition and facilitation. In R. R. Miller \& N. E. Spear (Eds.), Information processing in animals: Conditioned inhibition (pp. 299-326). Hillsdale, NJ: Erlbaum.

RESCORLA, R. A. (1986a). Extinction of facilitation. Journal of Experimental Psychology: Animal Behavior Processes, 12, 16-24.

RESCORLA, R. A. (1986b). Facilitation and excitation. Journal of Experimental Psychology: Animal Behavior Processes, 12, 325-332.

RESCORLA, R. A. (1987). Facilitation and inhibition. Journal of Experimental Psychology: Animal Behavior Processes, 13, 250-259.

Rescorla, R. A. (1988). Facilitation based on inhibition. Animal Learning \& Behavior, 16, 169-176.

RESCORLA, R. A. (1989). Simultaneous and sequential conditioned inhibition in autoshaping. Quarterly Journal of Experimental Psychology, 41B, 275-286.

RESCORLA, R. A. (1991a). Combinations of modulators trained with the same and different target stimuli. Animal Learning \& Behavior, 19, $355-360$

REsCORLA, R. A. (1991b). Separate reinforcement can enhance the effectiveness of modulators. Journal of Experimental Psychology: Animal Behavior Processes, 17, 259-269.

RESCORLA, R. A. (1991c). Transfer of inhibition and facilitation mediated by the original target stimulus. Animal Learning \& Behavior, $19,65-70$.

Rescorla, R. A., \& Holland, P. C. (1977). Associations in Pavlovian conditioned inhibition. Learning \& Motivation, 8, 429-447.

ResCoRla, R. A., \& WAGNer, A. R. (1972). A theory of Pavlovian conditioning: Variations in the effectiveness of reinforcement and nonreinforcement. In A. H. Black \& W. F. Prokasy (Eds.), Classical conditioning II: Current research and theory (pp. 64-99). New York: Appleton-Century-Crofts.
RoBBins, S. J. (1990). Mechanisms underlying spontaneous recovery in autoshaping. Journal of Experimental Psychology: Animal Behavior Processes, 16, 235-249.

Ross, R. T. (1983). Relationships between the determinants of performance in serial feature positive discriminations. Journal of Experimental Psychology: Animal Behavior Processes, 9, 349-373.

Ross, R. T., \& Holland, P. C. (1981). Conditioning of simultaneous and serial feature-positive discriminations. Animal Learning \& Behavior, 9, 293-303.

Ross, R. T., \& Holland, P. C. (1982). Serial positive patterning: Implications for "occasion setting." Bulletin of the Psychonomic Society, 19, 159-162.

Ross, R. T., \& LoLoRDo, V. M. (1986). Blocking during serial featurepositive discriminations: Associative versus occasion-setting functions. Journal of Experimental Psychology: Animal Behavior Processes, 12, 315-324.

SKINNER, B. F. (1938). The behavior of organisms. New York: AppletonCentury-Crofts.

SWARTZENTRUBER, D. (1991). Blocking between occasion setters and contextual stimuli. Journal of Experimental Psychology: Animal Behavior Processes, 17, 163-173.

SwarTZENTRUBER, D. (1993). Transfer of contextual control across similarly trained conditioned stimuli. Animal Learning \& Behavior, 21, 14-22.

Swartzentruber, D., \& Bouton, M. E. (1988). Transfer of positive contextual control across different conditioned stimuli. Bulletin of the Psychonomic Society, 26, 569-572.

Swartzentruber, D., \& Rescorla, R. A. (1994). Modulation of trained and extinguished stimuli by facilitators and inhibitors. Animal Learning \& Behavior, 22, 309-316.

TAIT, R. W., \& SAladin, M. E. (1986). Concurrent development of excitatory and inhibitory associations during backward conditioning. Animal Learning \& Behavior, 14, 133-137.

WAGNER, A. R., \& BRANDON, S. E. (1989). Evolution of a structured connectionist model of Pavlovian conditioning (AESOP). In S. B. Klein \& R. R. Mowrer (Eds.), Contemporary learning theoriesPavlovian conditioning and the status of traditional learning theory (pp. 149-189). Hillsdale, NJ: Erlbaum.

Williams, D. A., \& Overmier, J. B. (1988). Some types of conditioned inhibitors carry collateral excitatory associations. Learning \& $\mathrm{Mo}^{-}$ tivation, 19, 345-368.

Williams, D. A., Overmier, J. B., \& LoLordo, V. M. (1992). A reevaluation of Rescorla's early dictums about Pavlovian conditioned inhibition. Psychological Bulletin, 111, 274-290.

Williams, D. A., Travis, G. M., \& Overmier, J. B. (1986). Withincompound associations modulate the relative effectiveness of differential and Pavlovian conditioned inhibition procedures. Journal of Experimental Psychology: Animal Behavior Processes, 12, 351362.

Wilson, P. N., \& Pearce, J. M. (1989). A role for stimulus generalization in conditional discrimination learning. Quarterly Journal of Experimental Psychology, 41B, 243-273.

WILSON, P. N., \& PEARCE, J. M. (1990). Selective transfer of responding in conditional discriminations. Quarterly Journal of Experimental Psychology, 42B, 41-58.

(Manuscript received January 11, 1994; revision accepted for publication September 13, 1994.) 